\title{
1 A GPU-accelerated cortical neural network model for visually \\ 2 guided robot navigation
}

3 Michael Beyeler ${ }^{1}$, Nicolas Oros ${ }^{2}$, NikilDutt ${ }^{1,2}$, and Jeffrey L. Krichmar ${ }^{1,2}$

4 1Department of Computer Science, University of California Irvine, Irvine, CA, USA

$5 \quad$ 2Department of Cognitive Sciences, University of California Irvine, Irvine, CA, USA

9 Keywords:

10 Obstacle avoidance;spiking neural network; robot navigation;motion energy;MT; GPU.

14 Correspondence:

15 Michael Beyeler

16 University of California, Irvine

17 Department of Computer Science

18 Irvine, CA 92697

19 mbeyeler@uci.edu

20 Office of Jeff Krichmar: (949) 824-5888 


\section{1. Abstract}

2 Humans and other terrestrial animals use vision to traverse novel cluttered 3 environments with apparent ease.On one hand, although much is known about the

4 behavioral dynamics of steering in humans, it remains unclear how relevant perceptual 5 variables might be represented in the brain. On the other hand, although a wealth of data 6 exists about the neural circuitry that is concerned with the perception of self-motion 7 variables such as the current direction of travel, little research has been devoted to 8 investigating how this neural circuitry may relate to active steering control. Here we 9 present a cortical neural network model for visually guided navigation that has been 10 embodied on a physical robot exploring a real-world environment. The model includes a 11 rate based motion energy model for area V1, and a spiking neural network model for 12 cortical area MT. The model generates a cortical representation of optic flow, determines 13 the position of objects based on motion discontinuities, and combines these signals with 14 the representation of a goal location to produce motor commands that successfully steer 15 the robot around obstacles towards the goal. The model producesrobot trajectories that 16 closely match human behavioral data. This study demonstrates how neural signals in a 17 model of cortical area MT might provide sufficient motion information to steer a physical 18 robot on human-like paths around obstacles in a real-world environment, and exemplifies 19 the importance of embodiment, as behavior is deeply coupled not only with the underlying 20 model of brain function, but also with the anatomical constraints of the physical body it 21 controls. 


\section{Introduction}

23 Animals are capable of traversing novel cluttered environments with apparent ease. In

24 particular, terrestrial mammals use vision to routinely scan the environment, avoid 25 obstacles, and approach goals. Such locomotor tasks require the simultaneous integration 26 of perceptual variables within the visual scene in order to rapidly execute a series of finely 27 tuned motor commands. Although there have been studies on the behavioral dynamics of 28 steering in humans (Fajen \& Warren, 2003; Wilkie \& Wann, 2003), it remains unclear how 29 the perceptual variables highlighted therein might be represented in the brain. Only 30 recently did researchers begin to ask whether individual behavioral performance is 31 reflected in specific cortical regions (Billington, Wilkie, \& Wann, 2013; Field, Wilkie, \&

32 Wann, 2007). Despite a wealth of data regarding the neural circuitry concerned with the 33 perception of self-motion variables such as the current direction of travel ("heading") 34 (Britten \& van Wezel, 1998; Duffy \& Wurtz, 1997; Gu, Watkins, Angelaki, \& DeAngelis, 35 2006)or the perceived position and speed of objects(Eifuku \& Wurtz, 1998; Tanaka, Sugita, 36 Moriya, \& Saito, 1993), little research has been devoted to investigating how this neural 37 circuitry may relate to active steering control.

38 Visually guided navigation has traditionally attracted much attention from the domains 39 of both vision and control, producing countless computational models with excellent 40 navigation and localization performance (for a recent survey see Bonin-Font, Ortiz, and 41 Oliver (2008)). However, the majority of these models have taken a computer science or 42 engineering approach, without regard to biological or psychophysical fidelity. To date, only 43 a handful of neural network models have been able to explain the trajectories taken by 44 humans to steer around stationary objects toward a goal (Browning, Grossberg, \& Mingolla, 45 2009; Elder, Grossberg, \& Mingolla, 2009), and none of them have been tested in real-world 46 environments. The real world is the ultimate test bench for a model that is trying to link 47 perception to action, because even carefully devised simulated experiments typically fail to 48 transfer to real-world settings. Real environments are rich, multimodal, and noisy; an 49 artificial design of such an environment would be computationally intensive and difficult to 50 simulate (Krichmar \& Edelman, 2006). Yet real-world integration is often prohibited due to 51 engineering requirements, programming intricacies, and the sheer computational cost that 
52 come with large-scale biological models. Instead, such models often find application only in 53 constrained or virtual environments, which may severely limit their explanatory power 54 when it comes to generalizing findings to real-world conditions.

55 In this paper, we present a cortical neural network model for visually guided navigation 56 that has been embodied on a physical robot exploring a real-world environment (see 57 Figure 1). All experiments were performed by "Le Carl", an Android Based Robotics (ABR) 58 platform (Oros \& Krichmar, 2013b)constructed from the chassis of an R/C car. An Android 59 phone, mounted on the robot, was used to stream image frames while the robot was 60 traversing a hallway of an office building.The images were sent via WiFi to a remote 61 machine hosting the cortical neural network model. The neural network model generated a 62 cortical representation of dense optic flow and determined the position of objects based on 63 motion discontinuities. These signals then interacted with the representation of a goal 64 location to produce motor commands that successfully steered the robot around obstacles 65 towards the goal.The neural network model produced behavioral trajectories that not only 66 closely matched human behavioral data (Fajen \& Warren, 2003), but were also surprisingly 67 robust.

68 To the best of our knowledge, this is the first cortical neural network model 69 demonstrating human-like smooth paths in real-time in a real-world environment. The 70 present model is inspired by the more comprehensiveSTARS model (Elder, et al., 2009)and 71 its successor, the ViSTARS model (Browning, et al., 2009). All three models share some 72 similarities, such as the use of cortical motion processing mechanisms in area MT to induce 73 repeller-like obstacle avoidance behavior. Notable differences are that the present model 74 uses a widely accepted model of cortical motion perception (Simoncelli \& Heeger, 1998) to 75 generate neural motion signals, that it combines these signals with a much simpler steering 76 control scheme, and that it does not attempt to model self-motion processing in higher77 order parietal areas.

78 It should also be noted that area MT of the present model, which was responsible for 79 generating the motion output, was composed of thousands of simulated biologically 80 realistic excitatory and inhibitory spiking neurons (Izhikevich, 2003) withsynaptic 81 conductances for AMPA, NMDA, GABAa, and GABAb. Building neurorobotics agents whose 
82 behavior depends on a spiking neuron model that respects the detailedtemporal dynamics

83 of neuronal and synaptic integration of biological neurons may facilitate the investigation

84 of how biologically plausible computation can give rise to adaptive behavior in space and

85 time. In addition, spiking neurons are compatible with recent neuromorphic architectures

86 (Boahen, 2006; Cassidy, et al., 2014; Khan, et al., 2008; Schemmel, et al., 2010; Srinivasa \&

87 Cruz-Albrecht, 2012) and neuromorphic sensors (Lichtsteiner, Posch, \& Delbruck, 2008;

88 Liu, van Schaik, Minch, \& Delbruck, 2010; Wen \& Boahen, 2009). Thus, developing complex

89 spiking networks that display cognitive functions or learn behavioral abilities through

90 autonomous interaction may also represent an important step toward realizing functional

91 large-scale networks on neuromorphic hardware.

92 Overall, this study demonstrates how neural signals in a cortical model of visual motion

93 perceptioncan respond adaptively to natural scenes, and how the generated motion signals

94 are sufficient to steer a physical robot on human-like paths around obstacles. In addition,

95 this study exemplifies the importance of embodiment for the validation of brain-inspired

96 models, as behavior is deeply coupled not only with the underlying model of brain function,

97 but also with the anatomical constraints of the physical body it controls. 


\section{Methods}

99

100

101

102

103

104

105

106

107

108

109

110

111

112

113

114

115

116

117

118

119

120

121

122

123

124

125

126

\subsection{The robotic platform}

In order to engineer a system capable of real-time execution and real-world integration of large-scale biological models, we needed to address several technical challenges as outlined below.

First, the Android Based Robotics (ABR) framework was used as a flexible and inexpensive open-source robotics platform. In specific, we made use of the "Le Carl" robot, an ABR platform (Oros \& Krichmar, 2012, 2013a, 2013b) constructed from the chassis of an R/C car (see Figure 2). The main controller of the platform was an Android cell phone (Samsung Galaxy S3), which mediated communication via Bluetooth to an IOIO electronics board (SparkFun Electronics, http://www.sparkfun.com), which in turn sent PWM commands to the actuators and speed controllers of the R/C car. Since today's smartphones are equipped with a number of sensors and processors, it is possible to execute computationally demanding software based on real-time sensory data directly on the phone. The first neurorobotics study to utilize the ABR platform implemented a neural network based on neuromodulated attentional pathways to perform a reversal learning task by fusing a number of sensory inputs such as GPS location, a compass reading, and the values of on-board IR sensors (Oros \& Krichmar, 2012). However, the complexity and sheer size of the present model did not allow for on-board processing, and instead required hosting the computationally expensive components on a remote machine.

Second, an Android app (labeled "ABR client" in Figure 2) was developed to allow the collection of sensory data and communication with a remote machine (labeled "ABR server" in Figure 2) via WiFi and 3G. The only sensor used in the present study was the Android phone's camera, which collected 320x240 pixel images while the robot was behaving. These images were then sent via UDP to the ABR server, at a rate of roughly 20 frames per second. The neural network processed the stream of incoming images in realtime and generated motor commands, which were received by the ABR client app running onthe phone via TCP. The ABR client communicated motor commands directly to the corresponding actuators and speed controllers of the R/C car via the IOIO board. 
127 Third, we developed a Qt software interface that allowed integration of the ABR server 128 with different $\mathrm{C} / \mathrm{C}++$ libraries such as CUDA, OpenCV, and the spiking neural network 129 (SNN) simulator CARLsim (Beyeler, Carlson, Shuo-Chou, Dutt, \& Krichmar, 2015;

130 Nageswaran, Dutt, Krichmar, Nicolau, \& Veidenbaum, 2009; Richert, Nageswaran, Dutt, \& 131 Krichmar, 2011). In order to adhere to the real-time constraints of the system, all 132 computationally intensive parts of the model were accelerated on a GPU (i.e., a single 133 NVIDIA GTX 780 with 3 GB of memory) using the CUDA programming framework.

\section{$134 \quad 3.2$ The cortical neural network model}

135 The high-levelarchitecture of the cortical neural network model is shown in Figure 2.

136 The model wasbased on a cortical model of V1 and MT that was previously shown to 137 produce direction and speed tuning curves comparable to electrophysiological results in 138 response to synthetic visual stimuli such as sinusoidal gratings and plaids (Beyeler, Richert, 139 Dutt, \& Krichmar, 2014). The model used an efficient GPU implementation of the motion 140 energy model (Simoncelli \& Heeger, 1998) to generate cortical representations of motion in 141 a model of the primary visual cortex (V1). Spiking neurons in a model of the middle 142 temporal (MT) area then located nearby obstacles by means of motion discontinuities 143 (labeled "Obstacle component" in Figure 2). The MT motion signals projected toa simulated 144 posterior parietal cortex (PPC), where they interacted with the representation of a goal 145 location (labeled "Goal component" in Figure 2) to produce motor commands to steer the 146 robot around obstacles towards a goal. The following subsections will explain the model in 147 detail.

\section{$148 \quad 3.2 .1$ Visual input}

149 Inputs to the model were provided by the built-in camera of an Android phone 150 (Samsung Galaxy S3) mounted on the ABR platform. The phone took 320x240 pixel 151 snapshots of the environment at a rate of roughly 20 frames per second. These frames 152 entered the model in two ways: First, mimicking properties of visual processing in the 153 magnocellular pathway, a grayscale, down-scaled version of the lower half of the frame 154 (80x30 pixels) was sent to the network processing visual motion (obstacle component). 155 This pathway consisted of a preprocessing stage as well as a model of V1, MT, and the PPC. 
156 Second, mimicking properties of visual processing in the parvocellular pathway, the

157 originally collected RGB frame (320x240 pixels) was sent to a model component concerned

158 with locating a visually salient target in the scene (goal component). Because a neural

159 implementation of this pathway was considered out of scope for the present study, the goal

160 object was located using OpenCV-based segmentation in HSV color space. The location of

161 the goal in the visual field was then communicated to the PPC.

\section{$162 \quad 3.2 .2$ Preprocessing}

163 The first stage of the obstacle component pathway (labeled "pre" in Figure 2) enhanced 164 contrast and normalized the input imageusing OpenCV's standard implementation of 165 contrast-limited adaptive histogram equalization (CLAHE). The processed framewas then 166 sent to the V1 stage.

\section{$167 \quad 3.2 .3$ Primary visual cortex (V1)}

168 The second stage of the obstacle component pathway (labeled "V1" in Figure 2) used the 169 motion energy model to implement model neurons that responded to a preferred direction 170 and speed of motion (Simoncelli \& Heeger, 1998). In short, the motion energy model used a 171 bank of linear space-time oriented filters to model the receptive field of directionally 172 selective simple cells in V1. The filter responses were half-rectified, squared, and 173 normalized within a large spatial Gaussian envelope. The output of this stage was 174 equivalent to rate-based activity of V1 complex cells, whose responses were computed as 175 local weighted averages of simple cell responses in a Gaussian neighborhood of $\sigma_{V 1 c}=1.6$ 176 pixels (see Figure 3). The resulting size of the receptive fields wasroughly one degreeof the 177 visual field (considering that the horizontal field of view of a Samsung Galaxy S3 is roughly 17860 degrees), which is in agreement with electrophysiological evidence from recordings in 179 macaque V1 (Freeman \& Simoncelli, 2011).V1 complex cells responded to eight different 180 directions of motion (in 45 degree increments) at a speed of 1.5 pixels per frame. We 181 interpreted these activity values as neuronal mean firing rates, which were scaled to match 182 the contrast sensitivity function of V1 complex cells (see Beyeler et al. (2014)). Based on 183 these mean firing rates we then generated Poisson spike trains (of 50 ms duration), which 
184 served as the spiking input to Izhikevich neurons representing cells in area MT. More

185 information about the exact implementation can be found in Beyeler et al. (2014).

\section{$186 \quad 3.2 .4$ Middle temporal (MT) area}

187 The simulated area MT(labeled "MT" in Figure 2) consisted of 40,000 Izhikevich spiking 188 neurons and roughly 1,700,000 conductance-based synapses, which aimed to extract the 189 position and perceived size of any nearby obstacles by means of detecting motion 190 discontinuities. Neurons in MT received optic flow-like input from V1 cells, thus inheriting 191 their speed and direction preferences (Born \& Bradley, 2005). Their spatial receptive fields 192 had a circular center preferring motion in a particular direction, surrounded by a region 193 preferring motion in the anti-preferred direction (Allman, Miezin, \& McGuinness, 1985; 194 Born, 2000). These receptive fields were implemented as a difference of Gaussians: 195 Excitatory neurons in MT received input from Poisson spike generators in V1 in a narrow 196 spatial neighborhood ( $\sigma_{M T e}=6.0$ pixels) and from inhibitory neurons in MT in a 197 significantly larger spatial neighborhood $\left(\sigma_{M T i}=12.0\right.$ pixels; see Figure 3$)$, which are 198 comparable in size to receptive fields of neurons in layers 4 and 6 of macaque MT (Raiguel, 199 Van Hulle, Xiao, Marcar, \& Orban, 1995). The weights and connection probabilities scaled 200 with distance according to the Gaussian distribution. The maximum excitatory weight (at 201 the center of a Gaussian kernel) was 0.01 and the maximum inhibitory weight was 0.0015 202 (see (4) below). As a result of their intricate spatial receptive fields, excitatory neurons in

203 MT were maximally activated by motion discontinuities in the optic flow field, which is 204 thought to be of use for detecting object motion (Allman, et al., 1985; Bradley \& Andersen, 205 1998).Note that cortical cells with such a receptive field organization usually exhibit 206 different binocular disparity preferences in their center and surround regions (Bradley \& 207 Andersen, 1998). However, because the model had access to only a single camera, we were 208 unable to exploit disparityinformation (for more information please refer to Section 209 "Modellimitations").

210 All neurons in MT were modeled as Izhikevich spiking neurons (Izhikevich, 2003). The

211 Izhikevich model aims to reduce Hodgkin-Huxley-type neuronal models to a two212 dimensional system of ordinary differential equations, 


$$
\begin{gathered}
\frac{d v(t)}{d t}=0.04 v^{2}(t)+5 v(t)+140-u(t)+i_{\text {syn }}(t) \\
\frac{d u(t)}{d t}=a(b v(t)-u(t)) \\
v(v>30)=c \text { and } u(v>30)=u-d .
\end{gathered}
$$

213 Here, (1) described the membrane potential $v$ for a given synaptic current $i_{\text {syn }}$, whereas

214 (2) described a recovery variable $u$; the parameter $a$ was the rate constant of the recovery 215 variable, and $b$ described the sensitivity of the recovery variable to the subthreshold 216 fluctuations of the membrane potential. Whenever $v$ reached peak $\left(v_{\text {cutoff }}=+30\right)$, the 217 membrane potential was reset to $c$ and the value of the recovery variable was decreased by $218 d$ (see (3)). The inclusion of $u$ in the model allowed for the simulation of typical spike 219 patterns observed in biological neurons. The four parameters $a, b, c$, and $d$ can be set to 220 simulate different types of neurons. All excitatory neurons were modeled as regular spiking 221 (RS) neurons (class 1 excitable, $a=0.02, b=0.2, c=-65, d=8$ ), and all inhibitory 222 neurons were modeled as fast spiking (FS) neurons (class 2 excitable, $a=0.1, b=0.2$, $223 c=-65, d=2$ ) (Izhikevich, 2003).

224 Ionic currents were modeled as dynamic synaptic channels with zero rise time and 225 exponential decay:

$$
\frac{d g_{r}(t)}{d t}=-\frac{1}{\tau_{r}} g_{r}(t)+\eta_{r} w \sum_{i} \delta\left(t-t_{i}\right),
$$

226 where $\delta$ was the Dirac delta, the sum was over all presynaptic spikes arriving at time $t_{i}$, $227 w$ was the weight of the synapse (hand-selected to be 0.01 at the center of an excitatory 228 Gaussian kernel, and 0.0015 at the center of an inhibitory Gaussian kernel), $\tau_{r}$ was its 229 decay time constant, $\eta_{r}$ was a receptor-specific efficacy (or synaptic gain), and the 230 subscript $r$ denoted the receptor type; that is, AMPA (fast decay, $\tau_{\mathrm{AMPA}}=5 \mathrm{~ms}$ ), NMDA 231 (slow decay and voltage-dependent, $\tau_{\mathrm{NMDA}}=150 \mathrm{~ms}$ ), GABAa (fast decay, $\tau_{\mathrm{GABA}_{\mathrm{a}}}=6 \mathrm{~ms}$ ), 232 and GABAb (slow decay, $\tau_{\mathrm{GABA}_{\mathrm{a}}}=150 \mathrm{~ms}$ ). A spike arriving at a synapse that was post233 synaptically connected to an excitatory (inhibitory) neuron increased both $g_{\text {AMPA }}$ and $234 g_{\mathrm{NMDA}}\left(g_{\mathrm{GABA}_{\mathrm{a}}}\right.$ and $\left.g_{\mathrm{GABA}_{\mathrm{b}}}\right)$ with receptor-specific efficacy $\eta_{\mathrm{AMPA}}=1.5$ and $\eta_{\mathrm{NMDA}}=0.5$ $235\left(\eta_{\mathrm{GABA}_{\mathrm{a}}}=1.0\right.$ and $\left.\eta_{\mathrm{GABA}_{\mathrm{b}}}=1.0\right)$. Having $\eta_{\mathrm{AMPA}}>\eta_{\mathrm{NMDA}}$ agrees with experimental 
236 findings (Myme, Sugino, Turrigiano, \& Nelson, 2003) and allowed the network to quickly 237 react to changing sensory input. The total synaptic current $i_{\text {syn }}$ in (1) was then given by:

$$
\begin{aligned}
i_{\mathrm{Syn}}= & -g_{\mathrm{AMPA}}(v-0) \\
& -g_{\mathrm{NMDA}} \frac{\left(\frac{v+80}{60}\right)^{2}}{1+\left(\frac{v+80}{60}\right)^{2}}(v-0) \\
& -g_{\mathrm{GABA}_{\mathrm{a}}}(v+70) \\
& -g_{\mathrm{GABA}_{\mathrm{b}}}(v+90) .
\end{aligned}
$$

238 For more information on the exact implementation of the Izhikevich model, please refer 239 to the CARLsim 2.0 release paper (Richert, et al., 2011).

\section{$240 \quad 3.2 .5$ Posterior parietal cortex (PPC)}

241 The simulated PPC(labeled "PPCl" and "PPCr" in Figure 2) combined visual 242 representations of goal and obstacle information to produce a steering signal. The resulting 243 dynamics of the steering signal resembled the Balance Strategy, which is a simple control 244 law that aims to steer away from large sources of optic flow in the visual scene. For 245 example, honeybees use this control law to steer collision-free paths through even narrow 246 gaps by balancing the apparent speeds of motion of the images in their eyes (Srinivasan \& 247 Zhang, 1997). Interestingly, there is also some evidence for the Balance Strategy in 248 humans (Kountouriotis, et al., 2013).However, the present model differs in an important 249 way from the traditional Balance Strategy, in that it tries to balance the flow generated 250 from motion discontinuities in the visual field, which are thought to correspond to

251 obstacles in the scene, instead of balancing a conventional optic flow field. For more 252 information see Section "Neurophysiological evidence and model alternatives".

253 The steering signal took the form of a turning rate, $\dot{\theta}$ :

$$
\dot{\theta}=\hat{\theta}-\theta,
$$

254 which was derived from the difference between the robot's current angular orientation,

$255 \theta$, and an optimal angular orientation estimated by the cortical network, $\hat{\theta}$.The variable $\theta$ 256 was based on a copy of the current PWM signal sent to the servos of the robot (efference 257 copy), whereas the variable $\hat{\theta}$ was derived from the neural activation in PPC, as described 258 in (7) below. The resulting turning rate, $\dot{\theta}$, was then directly mapped into a PWM signal 
259 sent to the servos that controlled the steering of the robot.In order not to damage the 260 servos, we made sure that the computed instantaneous change in turning rate, $\ddot{\theta}$, never 261 exceeded a threshold (set at roughly $10 \%$ of the full range of PWM values). Steering with 262 the second derivative also contributed to the paths of the robot being smoother.

263 The cortical estimate of angular orientation was realized by separately summing optic 264 flow information from the left $\left(F_{\mathrm{L}}\right)$ and right halves $\left(F_{\mathrm{R}}\right)$ of the visual scene according to the 265 Balance Strategy, and combining these flow magnitudes with information about the target 266 location $\left(T_{\mathrm{L}}\right.$ and $\left.T_{\mathrm{R}}\right)$ weighed with a scaling factor $\alpha$ (set at 0.6 ):

$$
\hat{\theta} \sim \frac{F_{\mathrm{L}}-F_{\mathrm{R}}+\alpha\left(T_{\mathrm{R}}-T_{\mathrm{L}}\right)}{F_{\mathrm{L}}+F_{\mathrm{R}}+\alpha\left(T_{\mathrm{L}}+T_{\mathrm{R}}\right)} .
$$

267 Here, the total optic flow in the left $\left(F_{\mathrm{L}}\right)$ and right $\left(F_{\mathrm{R}}\right)$ halves was computed as follows:

$$
\begin{aligned}
& F_{\mathrm{L}}=\sum_{\theta} \sum_{y=0}^{R-1} \sum_{x=0}^{C / 2-1}\left\|r_{\theta}(x, y) e_{\theta}\right\|, \\
& F_{\mathrm{R}}=\sum_{\theta} \sum_{y=0}^{R-1} \sum_{x=C / 2}^{C-1}\left\|r_{\theta}(x, y) e_{\theta}\right\|,
\end{aligned}
$$

268 where $R$ is the number of rows in the image, $C$ is the number of columns in the image, $269\|\cdot\|$ denotes the Euclidean 2-norm. Depending on the experiment, $r_{\theta}(x, y)$ is the firing rate of 270 either an MT or a V1 neuron that is selective to direction of motion $\theta$ at spatial location $271(x, y)$, and $e_{\theta}$ is a unit vector pointing in the direction of $\theta$.

272 The target location was represented as a two-dimensional blob of activity centered on 273 the target's center of mass $\left(x_{G}, y_{G}\right)$. If the target was located in the left half of the image, it 274 contributed to a term $T_{\mathrm{L}}$ :

$$
T_{\mathrm{L}}=\alpha A_{\mathrm{G}} \sum_{y=0}^{R-1} \sum_{x=0}^{C / 2-1} e^{-\frac{\left(x-x_{\mathrm{G}}\right)^{2}+\left(y-y_{\mathrm{G}}\right)^{2}}{2 \sigma_{\mathrm{G}}^{2}}},
$$

275 and if it was located in the right half of the image, it contributed to a term $T_{\mathrm{R}}$ :

$$
T_{\mathrm{R}}=\alpha A_{\mathrm{G}} \sum_{y=0}^{R-1} \sum_{x=C / 2}^{C-1} e^{-\frac{\left(x-x_{\mathrm{G}}\right)^{2}+\left(y-y_{\mathrm{G}}\right)^{2}}{2 \sigma_{\mathrm{G}}^{2}}},
$$

276 where $\alpha=0.6, \sigma_{G}=0.2 C$, and $A_{\mathrm{G}}$ was the perceived area of the target, which was 277 determined with OpenCV using color blob detection. This allowed the target contribution 
278 to increase with target size, which we assumed to scale inversely with target distance (also 279 compare Section "Modellimitations").Note that if the target was perfectly centered in the 280 visual field, it contributed equally to $T_{\mathrm{L}}$ and $T_{\mathrm{R}}$. Also, note that the contribution of the target 281 component from the right half of the image, $T_{R}$, to $\theta$ was positive, whereas the contribution 282 of the obstacle component $F_{\mathrm{R}}$ was negative. This led to the target component exhibiting an 283 attractor-like quality in the steering dynamics, whereas the goal component exhibited a 284 repeller-like quality. 


\subsection{Experimental setup}

In order to investigate whether the motion estimates in the simulated MT were sufficient for human-like steering performance, the model was tested on a reactive navigation task in the hallway of the University of California, Irvine Social and Behavioral Sciences Gateway, where our laboratory is located(see Figure 1). This setup provided a relatively narrow yet highly structured environment with multiple sources of artificial

292 lighting, thus challenging the cortical model to deal with real-world obstacles as well as to quickly react to changing illumination conditions.

Analogous to the behavioral paradigm of Fajen and Warren (2003), the robot's goal was to steer around an obstacle placed in its way (i.e., a recycle bin) in order to reach a distant goal (i.e., a yellow foam ball). The obstacle was placed between the location of the target and the robot's initial position, at three different angles (off-set by one, four, and eight degrees to the left of the robot's initial view $)$ and different distances $(2.5 \mathrm{~m}, 3 \mathrm{~m}$, and $3.5 \mathrm{~m}$ from the robot's initial position). For each of these configurations we ran a total of five trials, during which we collected both behavioral and simulated neural data. An example setup is shown in Figure 4A. At the beginning of each trial, the robot was placed in the same initial position, directly facing the target. The robot would then quickly accelerate to a maximum speed of roughly $1 \mathrm{~m} / \mathrm{s}$. Speed was then held constant until the robot had

304 navigated to the vicinity of the goal (roughly $0.5 \mathrm{~m}$ apart), at which point the trial was 305 ended through manual intervention.

306 An over-head camera was used to monitor the robot's path throughout each trial (see 307 Figure 4B). The camera was mounted on the wall such that it overlooked the hallway, and 308 was used to track the position of a large green marker attached to the rear of the robot 309 (best seen in Figure 1). Using a perspective transformation, a 320x240 pixel image (taken 310 every $100 \mathrm{~ms}$ ) was converted into a birds-eye view of the scene (see Figure 4C). We used 311 the standard OpenCV implementation of this transformation and fine-tuned the parameters

312 for the specific internal parameters of the camera. Although three-dimensional objects will 313 appear distorted (as is evident in Figure 4C), flat objects lying on the ground plane (i.e., the 314 hallway floor) will be drawn to scale. This allowed us to detect and track the green 
315 markeron the robot directly in the transformed image (Figure 4C), in which the size of the

316 marker does not depend on viewing distance (as opposed to Figure 4B). Color blob

317 detection was applied to find the marker in each frame, and the marker's location was used

318 to infer the robot's location. In order to aid tracking of the marker, we added temporal

319 constraints between pairs of subsequent frames for outlier rejection. This procedure not

320 only allowed us to automatically record the robot's location at any given time, but also

321 allowed direct comparison of the robot's behavioral results with human psychophysics

322 data.

\section{$323 \quad 4.2$ Behavioral results}

324 Fajen and Warren (2003) studied how humans walk around obstacles towards a 325 stationary goal, and developed a model to explain the steering trajectories of the study's 326 participants. Their work revealed that accurate steering trajectories can be obtained 327 directly from the scene geometry, namely the distance and angles to the goal and obstacles. 328 Using their behavioral model, we calculated "ground truth" paths for the scene geometry of 329 our experimental setup, and compared them to steering trajectories generated by the 330 robot's cortical neural network model.In order to assess the contribution of motion 331 processing in MT we conducted two sets of experiments: one where steering commands 332 were based solely on V1 activity, and one where steering commands were based on MT 333 activity, by adjusting $r_{\theta}(x, y)$ in (8) and (9).

334 Figure 5illustrates the robot's path around a single obstacle (recycle bin, ' $O$ ') towards a 335 visually salient goal (yellow foam ball, ' $\mathrm{X}$ ') for five different scene geometries, analogous to 336 Figure 10 in Fajen \& Warren (2003). Results are shown for steering with V1 (blue) as well 337 as for steering with MT (red). In the first three setups, the obstacle was placed $3 \mathrm{~m}$ away 338 from the robot's initial location, off-set by eight (red), four (green), and one (blue) degrees 339 to the left of the robot's initial view. Two other setups were tested, in which the obstacle 340 distance was $3.5 \mathrm{~m}$ (yellow) and $2.5 \mathrm{~m}$ (magenta) at an angle of 4 degrees. The robot's 341 mean path from five experimental runs is shown as a thick solid line in each panel, and the 342 shaded region corresponds to the standard deviation. The black dashed lines correspond to 343 the "ground truth" paths calculated from the behavioral model by Fajen and Warren (2003) 
344 using the corresponding scene geometry and standard parameter values. Each of these five 345 obstacle courses wasrun five times for a total of 25 successful trials.

346 Steering paths were generally more accurate and more robust when steering with MT as 347 opposed to steering with V1. Paths generated from neural activity in MT not only closely 348 matchedhuman behavioral data, but they also were surprisingly robust. That is, the robot 349 successfully completed all 25 trials without hitting an obstacle, the walls of the hallway, or 350 missing the goal. In fact, the only setup that proved difficult for the robot had the obstacle 351 placed only $2.5 \mathrm{~m}$ away from the robot (rightmost panel in Figure 5). In this scenario, 352 "ground truth" data indicates that humans start veering to the right within the first second 353 of a trial, which seemed not enough time for the robot. In contrast, steering from neural 354 activity in V1 led to a total of 7 crashes, which involved the robot driving either into the 355 obstacle or the wall, upon which the trial was aborted and all collected data discarded. We 356 repeated the experiment until the robot successfully completed five trials per condition. 357 When steering from V1 neural activity, the robot tended to react more strongly to 358 behaviorally irrelevant stimuli, leading to more variability in the robot's trajectories.

359 For all five tested conditions we calculated the path area error (using the trapezoidal 360 rule for approximating the region under the graph) as well as the maximum distance the 361 robot's path deviated from the ground truth at any given time. Mean and standard 362 deviation of these data (averaged over five trials each)are summarized in Table 1. All path 363 errors were on the order of $10^{-1} \mathrm{~m}^{2}$, with errors generated from steering with MT 364 consistently smaller than errors generated from steering with V1. When the robot was 365 steering from signals in MT, trial-by-trial variations were on the order of $10^{-2} \mathrm{~m}^{2}$, 366 suggesting that under these circumstances the robot reliably exhibited human-like steering 367 behavior in all tested conditions. The maximum distance that the robot's path deviated 368 from the human-like path was on the order of $10 \mathrm{~cm}$, which is much smaller than the 369 obstacle width $(37 \mathrm{~cm})$. In contrast, steering with V1 often led to much larger deviations in 370 some cases. The best single-trial result was $0.084 \mathrm{~m}^{2}$ area error and $5 \mathrm{~cm}$ maximum 371 deviation on the $-1^{\circ}$ condition. These results suggest that in most trials the task could still 372 be performed even when steering with V1, but that motion processing in MT was critical 373 for reliability and accuracy, both of which are distinct qualities of human-like steering. 


\begin{tabular}{|c|c|c|c|c|c|}
\hline \multirow{2}{*}{$\begin{array}{l}\text { Obsta } \\
\text { cle }\end{array}$} & \multirow{2}{*}{$\begin{array}{c}\text { Obsta } \\
\text { distance }\end{array}$} & $\begin{array}{l}\text { cle angle } \\
\text { (m) }\end{array}$ & Area error $\left(\mathbf{m}^{2}\right)$ & \multicolumn{2}{c|}{ Maximum deviation (m) } \\
\cline { 3 - 6 } & & V1 & MT & V1 & MT \\
\hline 3 & $-1^{\circ}$ & $0.472 \pm 0.174$ & $0.193 \pm 0.080$ & $0.237 \pm 0.077$ & $0.112 \pm 0.045$ \\
\hline 3 & $-4^{\circ}$ & $0.407 \pm 0.097$ & $0.140 \pm 0.024$ & $0.193 \pm 0.052$ & $0.091 \pm 0.013$ \\
\hline 3 & $-8^{\circ}$ & $0.354 \pm 0.050$ & $0.170 \pm 0.055$ & $0.165 \pm 0.020$ & $0.102 \pm 0.026$ \\
\hline 3.5 & $-4^{\circ}$ & $0.332 \pm 0.110$ & $0.264 \pm 0.100$ & $0.169 \pm 0.039$ & $0.143 \pm 0.029$ \\
\hline 2.5 & $-4^{\circ}$ & $0.575 \pm 0.176$ & $0.319 \pm 0.093$ & $0.288 \pm 0.099$ & $0.193 \pm 0.056$ \\
\hline
\end{tabular}

Furthermore, these results are comparable to simulated data obtained with the STARS model, where the authors reported path area errors and maximum deviations on the same order of magnitude (see Table 2 in Elder, Grossberg, and Mingolla (2009)). Their best result was $0.097 \mathrm{~m}^{2}$ area error and $2.52 \mathrm{~cm}$ maximum deviation on a single trial of the $-2^{\circ}$ condition. However, the STARS model did not directly process visual input, nor was it tested in a real-world environment. The follow-up study to the STARS model (called ViSTARS) did not provide quantitative data on path errors in the same manner that would allow comparison here. Thus similar performance to the STARS model is assumed.

\subsection{Neural activity}

Figure 6 shows the activity of neurons in V1 (Panel A) and MT (Panel B) recorded in a single trial of the third setup in Figure 5 (blue). For the sake of clarity, only every eighth neuron in the population is shown over a time course of six seconds, which included both the obstacle avoidance and the goal approach. Neurons are organized according to their 392 motion $\left(0^{\circ}\right)$, IDs 300-599 mappedto upward-rightward motion at45, IDs 600-899 393 mapped to upward motion at 90 , IDs 900-1199 mapped to $135^{\circ}$, IDs 1200-1499 mapped 394 to $180^{\circ}$, IDs $1500-1799$ mapped to $225^{\circ}$, IDs $1800-2099$ mapped to $270^{\circ}$, and IDs $2100-$ 
2299 mapped to $315^{\circ}$.Panel A shows the spike trains generated from the rate-based

396 activity of V1 neurons using a Poisson spike generator. The firing of V1 neurons was broad

397 and imprecise in response to stimuli. In contrast, spiking responses of neurons in MT

398 (Panel B) were more selective, and sharper than V1.

399 As the robot approached the obstacle roughly from $1000 \mathrm{~ms}$ to $2500 \mathrm{~ms}$ of the trial

400 shown in Figure 6, neural activity in both V1 and MT steadily increased in both strength

401 and spatial extent. As activity in MT increased a repeller-like behavioral response was

402 triggered, which in turn introduced even more apparent motion on the retina (roughly

403 from $2200 \mathrm{~ms}$ to $3200 \mathrm{~ms}$ ). As soon as the obstacle moved out of the robot's view due to

404 successful avoidance (around $3200 \mathrm{~ms}$ ), activity in MT rapidly dropped off, causing the

405 robot's turning rate to momentarily decrease. With the goal still in view and growing in

406 size, turning rate was now increased with opposite sign (roughly from $3500 \mathrm{~ms}$ to

$4074500 \mathrm{~ms}$ ), leading to an attractor-like behavioral response that led the robot directly to the

408 goal. As the robot navigated to the goal (within a $0.5 \mathrm{~m}$ distance), the trial was ended

409 manually. Note that near the end of the trial (roughly from $4000 \mathrm{~ms}$ to $5000 \mathrm{~ms}$ ), neurons

410 in MT rightly perceived the goal object also as an obstacle, which is something the Fajen

411 and Warren model does not take into account. But, because the goal component of the

412 steering command grew more rapidly with size than the obstacle component, the robot

413 continued to approach the goal, rather than starting to avoid it.

414 Overall network activity and corresponding behavioral output is illustrated inFigure 7

415 and Figure 8. Each Panel summarizes the processing of a single visual input frame, which

416 corresponded to a time interval of $50 \mathrm{~ms}$. The indicated time intervals are aligned with the

417 neuronal traces presented in Figure 6. A frame received at time $t-50 \mathrm{~ms}$ led to a motor

418 response at time $t$. This sensorimotor delay was due to the fact that the SNN component of

419 the model needed to be executed for $50 \mathrm{~ms}$. Neural activity during these $50 \mathrm{~ms}$ is illustrated

420 as an optic flow field, overlaid on visual input, for both Poisson spike generators in V1 and

421 Izhikevich spiking neurons in MT. Here, every arrow represents the population vector of

422 the activity of eight neurons (selective to the eight directions of motion) coding for the

423 same spatial location. Note that, because these neurons were maximally selective to a

424 speed of 1.5 pixels per frame, vector length does not indicate velocity as is the case in 
425 aconventional optic flow field, but rather confidence in the direction estimate. For the sake 426 of clarity, only every fourth pixel location is visualized. The resulting turning rate, $\dot{\theta}$, which 427 was mapped to a PWM signal for the robot's servos, is illustrated using a sliding bar, where 428 the position of the triangle indicates the sign and magnitude of the turning rate. The small 429 horizontal line indicates $\dot{\theta}=0$. Note that the turning rate depended both on anstacle 430 term (from optic flow) and a goal term (from color blob detection) (see Section "Posterior 431 parietal cortex (PPC)").

432 Obstacle avoidance is illustrated in Figure 7. At the beginning of the trial (Panel A), the 433 image of the obstacle on the retina is too small to produce significant optic flow. Instead, V1 434 responds to arbitrary features of high contrast in the visual scene. Because these responses 435 were relatively low in magnitude and roughly uniformly distributed across the scene, MT 436 neurons successfully suppressed them, effectively treating them as noise. As a result, the 437 turning rate was near zero, informing the robot to steer straight ahead. In the following 438 second (Panels B-D) the obstacle as well as patterned regions in its vicinity generated an 439 increasingly uniform pattern of motion, leading to strong responses in MT and a strongly 440 positive turning rate. In turn, the initiated robot motion introduced even more apparent 441 motion on the retina, leading to a repeller-like behavioral response and successful obstacle 442 avoidance. Note that it is sometimes possible for the motion signals to slightly extend from 443 the object to neighboring contrast-rich regions, which is due to the strong motion pooling 444 in MT.

445 Goal-directed steering is illustrated in Figure 8. As soon as the obstacle moved out of the 446 robot's view due to successful avoidance (Panel A), activity in MT rapidly dropped off, 447 causing the robot's turning rate to momentarily decrease. With the goal still in view and 448 growing in size (Panels B-D), turning rate was now increased with opposite sign, 449 eventually overpowering the flow signals that would have instructed the robot to turn 450 away from the goal (Panel D), and instead leading to an attractor-like behavioral response 451 that drew the robot directly to the goal. As the robot navigated to the goal (within a $0.5 \mathrm{~m}$ 452 distance; following Panel D), the trial was ended manually. 
453 Overall these results demonstrate how a spiking model of MT can generate sufficient

454 information for the detection of motion boundaries, which can be used to steer a robot

455 around obstacles in a real-world environment.

$456 \quad 4.4$ Computational performance

457 The complete cortical model ran in real-time on a single GPU (NVIDIA GTX 780 with 4582304 CUDA cores and 3 GB of GDDR5 memory). In fact, during an average trial, most of the 459 visual frames were processed faster than real-time. In order for the network to satisfy the 460 real-time constraint, it must be able to process up to 20 frames per second, which was the 461 upper bound of the ABR client's frame rate. In practice, the effective frame rate might be 462 reduced due to UDP packet loss and network congestion (depending on the quality and 463 workload of the WiFi connection). In other words, the cortical model must process a single 464 frame in no more than $50 \mathrm{~ms}$.

465 The majority of the compute time was spent on neural processing in V1 and MT. Every $46650 \mathrm{~ms}$, model V1 computed a total of 201,600 filter responses (80x30 pixel image, 28 467 spatiotemporal filters at three different scales), taking up roughly $15 \mathrm{~ms}$ of execution time.

468 Model MT then calculated the temporal dynamics of 40,000 Izhikevich spiking neurons and 469 roughly 1,700,000 conductance-based synapses, which took roughly $25 \mathrm{~ms}$ of execution 470 time. During this time, a different thread performed color blob detection using OpenCV, 471 which allowed the total execution time to stay under $50 \mathrm{~ms}$. Compared to these 472 calculations, the time it took to perform CLAHE on the GPU was negligible. 


\section{Discussion}

We presented a neural network modeled after visual motion perception areas in the mammalian neocortex that is controlling a physical robot performing a visually-guided navigation task in the real world. The system described here builds upon our previous work on visual motion perception in large-scale spiking neural networks (Beyeler, et al., 2014). The prior system was tested only on synthetic visual stimuli to demonstrate neuronal tuning curves, whereas the present work demonstrates how perception may relate to action. As this is a first step toward an embodied neuromorphic system, we wanted to ensure that the model could handle real sensory input from the real world. Constructing an embodied model also ensured that the algorithm could handle noisy sensors, imprecise motor responses, as well as sensory and motor delays. The model generated a cortical representation of dense optic flow using thousands of interconnected spiking neurons, determined the position of objects based on motion discontinuities, and combined these signals with the representation of a goal location in order to calculate motor commands that successfully steered the robot around obstacles towards to the goal. Therefore, the present study demonstrates how cortical motion signals in a model of MT might relate to active steering control, and suggests that these signals might be sufficient to generate human-like trajectories through a cluttered hallway.This emergent behavior might not only be difficult to achieve in simulation, but also strengthens the claim that MT contributes to these smooth trajectories in natural settings. This finding exemplifies the importance of embodiment, as behavior is deeply coupled not only with the underlying model of brain function, but also with the anatomical constraints of the physical body.

495 The behavior of the robot was contingent on the quality of a cortical representation of 496 motion generated in a model of visual area MT. While it is generally assumed that vector497 based representations of retinal flow in both V1 and MT are highly accurate, modeling 498 work has suggested that the generation of highly accurate flow representations in complex 499 environments is challenging due to the aperture problem (Baloch \& Grossberg, 1997; 500 Bayerl \& Neumann, 2004; Chey, Grossberg, \& Mingolla, 1997; Simoncelli \& Heeger, 1998). 501 As a result, there is a degree of uncertainty in all retinal flow estimations. Nevertheless, the 502 present model is able to generate optic flow fields of sufficient quality to steer a physical 
503 robot on human-like trajectories through a cluttered hallway. Paramount to this 504 competence are the receptive fields of neurons in model MT, which are able to vastly 505 reduce ambiguities in the V1 motion estimates (see Figure 7 and Figure 8) and enhance 506 motion discontinuities through spatial pooling and directional opponent inhibition. As a 507 result, the robot is able to correctly infer the relative angle and position of nearby 508 obstacles, implicitly encoded by the spatial arrangement and overall magnitude of optic 509 flow, and produce steering commands that lead to successful avoidance.

510 It is interesting to consider whether there is a benefit to using spiking neurons instead of 511 rate based neurons. Under the present experimental conditions, the steering network may 512 have worked just as well with a model based on mean-firing rate neurons. However, there 513 is evidence that humans adjust their steering in response not only to spatial but also to 514 temporal asymmetries in the optic flow field (Duchon \& Warren, 2002; Kountouriotis, et al., 515 2013). Therefore, having at our exposala tested, embodied model of brain function that 516 respects the detailed temporal dynamics of neuronal and synaptic integration is likely to 517 benefit follow-up studies that aim to quantitatively investigate how such spatiotemporal 518 behavior can arise from neural circuitry. In addition, because the main functional 519 components of this system were event-driven spiking neurons, the model has the potential 520 to run on neuromorphic hardware, such as HRL (Srinivasa \& Cruz-Albrecht, 2012), IBM 521 True North (Cassidy, et al., 2014), NeuroGrid (Boahen, 2006), SpiNNaker (Khan, et al., 522 2008), andBrainScaleS (Schemmel, et al., 2010). Some of these platforms are now capable 523 of emulating neural circuits that contain more than a million neurons, at rates that are 524 significantly faster than real time. In addition, because spiking neurons communicate via

525 the AER protocol, the model could also be interfaced with an event-based, neuromorphic 526 vision sensor as a sensory front-end (Lichtsteiner, et al., 2008). Thus, developing complex 527 spiking networks that display cognitive functions or learn behavioral abilities through 528 autonomous interaction may also represent an important initial step into realizing 529 functional large-scale networkson neuromorphic hardware.

530 Additionally, we have developed software to further extend the functionality of the ABR 531 platform (Oros \& Krichmar, 2013b).A set of tools and interfaces exist that provide a 532 standard interface for non-experts to bring their models to the platform, ready for 
533 exploration of networked computation principles and applications. The fact that our 534 platform is open source, extensible, and affordablemakes ABR highly attractivefor 535 embodiment of brain-inspired models. For more information and software downloads, see 536 our website: www.socsci.uci.edu/ jkrichma/ABR.

\section{$537 \quad 5.1$ Neurophysiological evidence and model alternatives}

538 Human behavioral data suggests that visually-guided navigation might be based on 539 several possible perceptual variables, which can be flexibly selected and weighted 540 depending on the environmental constraints (Kountouriotis, et al., 2013; Morice, Francois, 541 Jacobs, \& Montagne, 2010). In the case of steering to a stationary goal, evidence suggests

542 that human subjects rely on both optic flow, such that one aligns the heading specified by 543 optic flow with the visual target (Gibson, 1958; Warren, Kay, Zosh, Duchon, \& Sahuc, 2001), 544 and egocentric direction, such that one aligns the locomotor axis with the egocentric 545 direction of the goal (Rushton, Harris, Lloyd, \& Wann, 1998). Optic flow tends to dominate 546 when there is sufficient visual surface structure in the scene (Li \& Warren, 2000; Warren, 547 et al., 2001; Wilkie \& Wann, 2003), whereas egocentric direction dominates when visual 548 structure is reduced (Rushton, et al., 1998; Rushton, Wen, \& Allison, 2002). Interestingly, 549 optic flow asymmetries are able to systematically bias the steering of human subjects,even

550 in the presence of explicit path information (Kountouriotis, et al., 2013). This finding may 551 hint at the possibility of a cortical analog to the Balance Strategy (Duchon \& Warren, 2002;

552 Srinivasan \& Zhang, 1997), which suggests that humans adjust their steering in response to 553 both spatial and temporal asymmetries in the optic flow field. However, the present model

554 differs in an important way from the traditional Balance Strategy, in that it does not try to

555 balance a conventional optic flow field. Instead, the model considers only signals generated 556 from motion discontinuities (due to motion processing in MT) in the balance equation, 557 which seems to have similar functional implications as the steering potential function from 558 Huang, Fajen, Fink, \& Warren (2006), generating a repeller signal that gets stronger the 559 closer the robot gets to the obstacle.

560 Visual self-motion is processed in a number of brain areas located in the 561 intraparietal (IPS) and cingulate sulci, including MST, the ventral intraparietal (VIP) region, 562 and the cingulate sulcus visual region (CSv) (Wall \& Smith, 2008). Lesions to the IPS lead to 
563 navigational impairments when retracting a journey shown from an egocentric 564 viewpoint (Seubert, Humphreys, Muller, \& Gramann, 2008). There is evidence that object 565 position and velocity might be encoded by a pathway that includes center-surround cells in

566 MT and cells in the ventral region of the medial superior temporal (MSTv) 567 area (Berezovskii \& Born, 2000; Duffy \& Wurtz, 1991b; Tanaka, et al., 1993). This object 568 information might then be combined with information about self-motion gathered in MST 569 and VIP from cues such as optic flow, eye rotations, and head movements (Andersen, 570 Essick, \& Siegel, 1985; Bradley, Maxwell, Andersen, Banks, \& Shenoy, 1996; Colby, 571 Duhamel, \& Goldberg, 1993; Duffy \& Wurtz, 1991a). How neural processing in these 572 regions could relate to steering control has been modeled in detail elsewhere (Browning, et 573 al., 2009; Elder, et al., 2009).

574 The present study demonstrates that it might not be necessary to explicitly model these 575 areas in order to explain human psychophysics data about visually-guided steering and 576 obstacle avoidance. In the case of a robot with a single stationary camera, there is no need 577 to calculate eye or head rotations. As a result of this, there is no need for coordinate 578 transformation, because retinotopic coordinates are equivalent to craniotopic coordinates. 579 These simplified anatomical constraints allow for gross simplification of the underlying 580 neural network model without restricting behavioral performance, at least under the 581 present experimental conditions.

\section{$582 \quad 5.2$ Modellimitations}

583 Although the present model is able to generate human-like steering paths for a variety of 584 experimental setups, it is does not attempt to explain how the direction of travel is 585 extracted from optic flow, how these signals are made independent of eye rotations and 586 head movements, and how these signals can be converted into a craniotopic reference 587 frame. However, these processes would need to be modeled if one were to consider a robot 588 that could freely move around its head (or its eye). A suitable future study could investigate 589 how these computations and transformations could be achieved for a robot that could

590 freely move around its head (or its eye), for example via a pan/tilt unit. The resulting 591 cortical model might not only reproduce the present behavioral results, but also lead to 592 robust behavior in more complicated experimental setups. 
593 As mentioned above, the behavioral model by Fajen and Warren (2003)computes a 594 steering trajectory using third-person information obtained directly from the scene 595 geometry, namely the distance and angles to the goal and obstacle. In contrast, our model 596 steers from a first-person view using active local sensing, which cannot directly compute 597 the distance and angle to the goal and obstacle. Instead, distance is implicitly represented 598 by neural activity, that is, the overall magnitude of the goal and activity related to obstacles. 599 This is based on the assumption that perceived object size scales inversely with distance. 600 While this assumption is generally true, it might not necessarily enable the model to 601 generalize to arbitrarily complex settings. For example, the model might try to avoid a large 602 but far away obstacle with the same dynamics as it would a small but close-by one. In this 603 case, an accurate estimate of object distance would be imperative.Also, in the current setup 604 it is sometimes possible that motion signals caused by the obstacle can extend into 605 contrast-rich neighboring regions, due to strong motion pooling in MT. A future study could 606 alleviate this issue by giving the robot a means to establish clearer boundaries between 607 objects and their surroundings, perhaps by modeling motion-form interactions (Baloch \& 608 Grossberg, 1997).

609 Binocular vision is an important source of depth perception that influences object 610 segmentation in depth (Xiao, Raiguel, Marcar, \& Orban, 1997) as well as the pre-planning 611 and on-line control of movement (Patla \& Vickers, 1997). Therefore it is possible that 612 binocular vision might significantly benefit the task at hand (Pauwels, Kruger, Lappe, 613 Worgotter, \& Van Hulle, 2010). A suitable follow-up study would thus be to quantify the 614 contribution of depth perception to the quality of stimulus responses in areas MT as well as 615 to the behavioral performance of the robotic agent. It is interesting to note that most MT 616 cells in the macaque receive balanced input from both eyes (Maunsell \& Van Essen, 1983), 617 but it turns out that neurons in (at least) macaque MT are not strongly tuned to motion in 618 depth; i.e., no units are truly activated for stimuli changing disparity, which would simulate 619 trajectories with components of motion toward or away from the animal (Maunsell \& Van 620 Essen, 1983). Therefore, extending the single camera version of the model by adding areas 621 important for segmenting a scene and recognizing objects (Baloch \& Grossberg, 1997), 622 would also improve planning trajectories through space. Because vergence is not a factor 
623 for distances on the order of meters, scene contributions from brain areas such as V4, MST,

624 and parietal cortex are probably important for long-range trajectory planning. Moreover, 625 these areas strongly interact with area MT.

626 Also, evidence suggests that humans use a different behavioral strategy when it comes 627 to intercepting moving targets (Fajen \& Warren, 2007). Having an agent steer toward a 628 moving target according to the present behavioral dynamics is likely to result in pursuit 629 behavior (as opposed to interception behavior), in which the agent always lags behind the 630 target. Thus we do not expect the model to generalize to moving targets. However, it might 631 be possible to extend the model to account for these scenarios. The superior parietal lobe 632 (SPL) might be involved in encoding future path information, such as the location of targets 633 and obstacles, which are indicative of impending changes in heading and using this for the 634 purpose of accurately timing motor responses (Billington, et al., 2013; Field, et al., 2007). 635 Computing and updating of object locations in space, which might be relevant especially for 636 moving targets, might be done by a network involving the precuneus and dorsal precentral 637 gyrus (Leichnetz, 2001; Parvizi, Van Hoesen, Buckwalter, \& Damasio, 2006). However, not 638 much is known about the neuronal representation of these signals.

\subsection{Practical implications}

640 The present work might be of interest to the neuroscientist, neurorobotics, and 641 neuromorphic engineering communities for the following reasons.

642 First, we have shown that the present approach is practical for studying the link 643 between neural circuitry and behavioral performance. The real-world consequences of 644 neural activity can be observed in real-time through visualization software and analyzed 645 off-line. The behavioral responses were comparable to psychophysical data and the 646 neuronal responses were comparable to neurophysiological data. We believe this to be a 647 powerful approach to studying models of neural circuitry in real-world environments.

648 Second, we have shown that the present system can handle neural network models of 649 non-trivial size. By making use of the CUDA programming framework, we were able to 650 accelerate computationally intensive parts of the model on a GPU. Every 50 ms, model V1 651 computed a total of 201,600 filter responses (80x30 pixel image, 28 spatiotemporal filters 652 at three different scales), and model MT calculated the temporal dynamics of 40,000 
653 Izhikevich spiking neurons and roughly 1,700,000 conductance-based synapses. If 654 necessary, the execution of the model could be sped up further, for example by parallelizing 655 processing in the goal and obstacle components of the model.

656 Third, implementing the entire model using spiking neurons would make the model 657 amenable to emulation on neuromorphic hardware (Cassidy, et al., 2014; Schemmel, et al., 658 2010; Srinivasa \& Cruz-Albrecht, 2012). This could enable the development of a self659 contained, fully autonomous neurorobotic platform that combines the algorithmic 660 advantages of the present model with the speed, efficiency, and scalability of neuromorphic 661 hardware. In addition, since spiking neural networks communicate via the AER protocol, 662 the model could be interfaced with an event-based, neuromorphic camera as a sensory 663 front-end (Lichtsteiner, et al., 2008). This would make it possible for the cortical network 664 to react more quickly (at least on the millisecond scale, but theoretically even on the 665 microsecond scale) to temporal changes in optic flow. It would also open the door for a 666 complete neuromorphic vision system that operates with low power and rapid responses.

667 Fourth, the system presented here is open source, extensible, and affordable. The 668 complete ABR source code is hosted on GitHub (www.github.com/UCI-ABR), and our 669 CARLsim simulator can be freely obtained from our website 670 (www.socsci.uci.edu/ jkrichma/CARLsim).The ABR framework can be combined with a 671 variety of $\mathrm{R} / \mathrm{C}$ based vehicles, sensors, actuators, and $\mathrm{C} / \mathrm{C}++$ based software components. 672 For example, it is straightforward to plug additional software components (such as image 673 transformation with OpenCV) into the client-server loop, or mount IR sensors on the robot 674 and read out the sensory values directly with the ABR client software. We also provide 675 online instructions and video tutorials to assemble the ABR platform, which has an 676 estimated cost of $\$ 200$ (excluding the phone). Because of this, we believe ABR to be an 677 attractive platform for students and researchers alike that will simplify both the 678 development of neurorobotics platforms as well as the study of how neural machinery can 679 be used to realize cognitive function. 


\section{6. Acknowledgments}

681 Supported by National Science Foundation award (IIS-0910710) and an award from 682 Qualcomm Technologies Incorporated. 
683

684

685

686

687

688

689

690

691

692

693

694

695

696

697

698

699

700

701

702

703

704

705

706

707

708

709

710

711

\section{References}

Allman, J., Miezin, F., \& McGuinness, E. (1985). Direction- and velocity-specific responses from beyond the classical receptive field in the middle temporal visual area (MT). Perception, 14, 105-126.

Andersen, R. A., Essick, G. K., \& Siegel, R. M. (1985). Encoding of spatial location by posterior parietal neurons. Science, 230, 456-458.

Baloch, A. A., \& Grossberg, S. (1997). A neural model of high-level motion processing: line motion and formotion dynamics. Vision Research, 37, 3037-3059.

Bayerl, P., \& Neumann, H. (2004). Disambiguating visual motion through contextual feedback modulation. Neural Comput, 16, 2041-2066.

Berezovskii, V. K., \& Born, R. T. (2000). Specificity of projections from wide-field and local motion-processing regions within the middle temporal visual area of the owl monkey. Journal of Neuroscience, 20, 1157-1169.

Beyeler, M., Carlson, K. D., Shuo-Chou, T., Dutt, N., \& Krichmar, J. L. (2015). CARLsim 3: A user-friendly and highly optimized library for the creation of neurobiologically detailed spiking neural networks. In IEEE International Joint Conference on Neural Networks. Killarney, Ireland.

Beyeler, M., Richert, M., Dutt, N. D., \& Krichmar, J. L. (2014). Efficient spiking neural network model of pattern motion selectivity in visual cortex. Neuroinformatics, 12, 435454.

Billington, J., Wilkie, R. M., \& Wann, J. P. (2013). Obstacle avoidance and smooth trajectory control: neural areas highlighted during improved locomotor performance. Front Behav Neurosci, 7, 9.

Boahen, K. (2006). Neurogrid: emulating a million neurons in the cortex. Conf Proc IEEE Eng Med Biol Soc, Suppl, 6702.

Bonin-Font, F., Ortiz, A., \& Oliver, G. (2008). Visual navigation for mobile robots: A survey. Journal of Intelligent \& Robotic Systems, 53, 263-296.

Born, R. T. (2000). Center-surround interactions in the middle temporal visual area of the owl monkey. Journal of Neurophysiology, 84, 2658-2669. 
Born, R. T., \& Bradley, D. C. (2005). Structure and function of visual area MT. Annual 713 Review of Neuroscience, 28, 157-189.

714 Bradley, D. C., \& Andersen, R. A. (1998). Center-surround antagonism based on disparity 715 in primate area MT. Journal of Neuroscience, 18, 7552-7565.

716 Bradley, D. C., Maxwell, M., Andersen, R. A., Banks, M. S., \& Shenoy, K. V. (1996). 717 Mechanisms of heading perception in primate visual cortex. Science, 273, 1544-1547.

718 Britten, K. H., \& van Wezel, R. J. (1998). Electrical microstimulation of cortical area MST 719 biases heading perception in monkeys. Nat Neurosci, 1, 59-63.

720 Browning, N. A., Grossberg, S., \& Mingolla, E. (2009). Cortical dynamics of navigation and 721 steering in natural scenes: Motion-based object segmentation, heading, and obstacle 722 avoidance. Neural Networks, 22, 1383-1398.

723 Cassidy, A. S., Alvarez-Icaza, R., Akopyan, F., Sawada, J., Arthur, J. V., Merolla, P. A., Datta, 724 P., Tallada, M. G., Taba, B., Andreopoulos, A., Amir, A., Esser, S. K., Kusnitz, J., Appuswamy, R., 725 Haymes, C., Brezzo, B., Moussalli, R., Bellofatto, R., Baks, C., Mastro, M., Schleupen, K., Cox, C. 726 E., Inoue, K., Millman, S., Imam, N., McQuinn, E., Nakamura, Y. Y., Vo, I., Guo, C., Nguyen, D., 727 Lekuch, S., Asaad, S., Friedman, D., Jackson, B. L., Flickner, M. D., Risk, W. P., Manohar, R., \& 728 Modha, D. S. (2014). Real-time scalable cortical computing at 46 giga-synaptic OPS/watt 729 with $\sim 100 \times$ speedup in time-to-solution and $\sim 100,000 \times$ reduction in energy-to-solution. In 730 Proceedings of the International Conference for High Performance Computing, Networking, 731 Storage and Analysis (pp. 27-38). New Orleans, Louisana: IEEE Press.

732 Chey, J., Grossberg, S., \& Mingolla, E. (1997). Neural dynamics of motion grouping: from 733 aperture ambiguity to object speed and direction. Journal of the Optical Society of America 734 a-Optics Image Science and Vision, 14, 2570-2594.

735 Colby, C. L., Duhamel, J. R., \& Goldberg, M. E. (1993). Ventral intraparietal area of the 736 macaque: anatomic location and visual response properties. Journal of Neurophysiology, 69, $737 \quad 902-914$.

738 Duchon, A. P., \& Warren, W. H., Jr. (2002). A visual equalization strategy for locomotor 739 control: of honeybees, robots, and humans. Psychol Sci, 13, 272-278. 
740 Duffy, C. J., \& Wurtz, R. H. (1991a). Sensitivity of MST neurons to optic flow stimuli. I. A 741 continuum of response selectivity to large-field stimuli. Journal of Neurophysiology, 65, $742 \quad 1329-1345$.

743 Duffy, C. J., \& Wurtz, R. H. (1991b). Sensitivity of MST neurons to optic flow stimuli. II. 744 Mechanisms of response selectivity revealed by small-field stimuli. Journal of 745 Neurophysiology, 65, 1346-1359.

746 Duffy, C. J., \& Wurtz, R. H. (1997). Planar directional contributions to optic flow 747 responses in MST neurons. Journal of Neurophysiology, 77, 782-796.

748 Eifuku, S., \& Wurtz, R. H. (1998). Response to motion in extrastriate area MSTl: center749 surround interactions. Journal of Neurophysiology, 80, 282-296.

750 Elder, D. M., Grossberg, S., \& Mingolla, E. (2009). A neural model of visually guided 751 steering, obstacle avoidance, and route selection. J Exp Psychol Hum Percept Perform, 35, 752 1501-1531.

753 Fajen, B. R., \& Warren, W. H. (2003). Behavioral dynamics of steering, obstacle 754 avoidance, and route selection. J Exp Psychol Hum Percept Perform, 29, 343-362.

755 Fajen, B. R., \& Warren, W. H. (2007). Behavioral dynamics of intercepting a moving 756 target. Exp Brain Res, 180, 303-319.

757 Field, D. T., Wilkie, R. M., \& Wann, J. P. (2007). Neural systems in the visual control of 758 steering. Journal of Neuroscience, 27, 8002-8010.

759 Freeman, J., \& Simoncelli, E. P. (2011). Metamers of the ventral stream. Nat Neurosci, 14, $760 \quad 1195-1201$.

761 Gibson, J. J. (1958). Visually controlled locomotion and visual orientation in animals. $\mathrm{BrJ}$ 762 Psychol, 49, 182-194.

763 Gu, Y., Watkins, P. V., Angelaki, D. E., \& DeAngelis, G. C. (2006). Visual and nonvisual 764 contributions to three-dimensional heading selectivity in the medial superior temporal 765 area. Journal of Neuroscience, 26, 73-85.

766 Huang, W. H., Fajen, B. R., Fink, J. R., \& Warren, W. H. (2006). Visual navigation and 767 obstacle avoidance using a steering potential function. Robotics and Autonomous Systems, $768 \quad 54,288-299$. 
Izhikevich, E. M. (2003). Simple model of spiking neurons. Ieee Transactions on Neural

770

771

772

773

774

775

776

777

778

779

780

781

782

783

784

785

786

787

788

789

790

791

792

793

794 795

796

797

798 Networks, 14, 1569-1572.

Khan, M., Lester, D., Plana, L., Rast, A., Jin, X., \& Painkras, E. (2008). SpiNNaker: Mapping neural networks onto a massively-parallel chip multiprocessor. In IEEE International Joint Conference on Neural Networks (pp. 2849-2856).

Kountouriotis, G. K., Shire, K. A., Mole, C. D., Gardner, P. H., Merat, N., \& Wilkie, R. M. (2013). Optic flow asymmetries bias high-speed steering along roads. J Vis, 13, 23.

Krichmar, J. L., \& Edelman, G. M. (2006). Principles underlying the construction of brainbased devices. In T. Kovacs \& J. A. R. Marshall (Eds.), Adaptation in Artificial and Biological Systems (Vol. 6, pp. 37-42). Bristol UK: Society for the Study of Artificial Intelligence and the Simulation of Behaviour.

Leichnetz, G. R. (2001). Connections of the medial posterior parietal cortex (area 7m) in the monkey. Anat Rec, 263, 215-236.

Li, L., \& Warren, W. H., Jr. (2000). Perception of heading during rotation: sufficiency of dense motion parallax and reference objects. Vision Research, 40, 3873-3894.

Lichtsteiner, P., Posch, C., \& Delbruck, T. (2008). A 128\&times; 128120 dB 15 \&\#956;s Latency Asynchronous Temporal Contrast Vision Sensor. Solid-State Circuits, IEEE Journal of, 43, 566-576.

Liu, S. C., van Schaik, A., Minch, B. A., \& Delbruck, T. (2010). Event-Based 64-Channel Binaural Silicon Cochlea with Q Enhancement Mechanisms. 2010 Ieee International Symposium on Circuits and Systems, 2027-2030.

Maunsell, J. H., \& Van Essen, D. C. (1983). Functional properties of neurons in middle temporal visual area of the macaque monkey. II. Binocular interactions and sensitivity to binocular disparity. Journal of Neurophysiology, 49, 1148-1167.

Morice, A. H., Francois, M., Jacobs, D. M., \& Montagne, G. (2010). Environmental constraints modify the way an interceptive action is controlled. Exp Brain Res, 202, 397411.

Myme, C. I., Sugino, K., Turrigiano, G. G., \& Nelson, S. B. (2003). The NMDA-to-AMPA ratio at synapses onto layer $2 / 3$ pyramidal neurons is conserved across prefrontal and visual cortices. Journal of Neurophysiology, 90, 771-779. 
Nageswaran, J. M., Dutt, N., Krichmar, J. L., Nicolau, A., \& Veidenbaum, A. V. (2009). A configurable simulation environment for the efficient simulation of large-scale spiking neural networks on graphics processors. Neural Networks, 22, 791-800.

Oros, N., \& Krichmar, J. L. (2012). Neuromodulation, attention and localization using a novel ANDROID robotic platform. In IEEE International Conference on Development and 804 Learning and Epigenetic Robotics. San Diego, CA.

805 Oros, N., \& Krichmar, J. L. (2013a). Android ${ }^{\mathrm{TM}}$ Based Robotics: Powerful, Flexible and 806 Inexpensive Robots for Hobbyists, Educators, Students and Researcher. In. Cognitive 807 Anteater Robotics Laboratory: University of California, Irvine.

808 Oros, N., \& Krichmar, J. L. (2013b). Smartphone Based Robotics: Powerful, Flexible and 809 Inexpensive Robots for Hobbyists, Educators, Students and Researchers. In CECS Technical 810 Report (pp. 13-16). Center for Embedded Computer Systems: University of California, 811 Irvine.

812 Parvizi, J., Van Hoesen, G. W., Buckwalter, J., \& Damasio, A. (2006). Neural connections of 813 the posteromedial cortex in the macaque. Proc Natl Acad Sci U S A, 103, 1563-1568.

814 Patla, A. E., \& Vickers, J. N. (1997). Where and when do we look as we approach and step 815 over an obstacle in the travel path? Neuroreport, 8, 3661-3665.

816 Pauwels, K., Kruger, N., Lappe, M., Worgotter, F., \& Van Hulle, M. M. (2010). A cortical 817 architecture on parallel hardware for motion processing in real time.J Vis, 10, 18.

818 Raiguel, S., Van Hulle, M. M., Xiao, D. K., Marcar, V. L., \& Orban, G. A. (1995). Shape and 819 spatial distribution of receptive fields and antagonistic motion surrounds in the middle 820 temporal area (V5) of the macaque. European Journal of Neuroscience, 7, 2064-2082.

821 Richert, M., Nageswaran, J. M., Dutt, N., \& Krichmar, J. L. (2011). An efficient simulation 822 environment for modeling large-scale cortical processing. Front Neuroinform, 5, 19.

823 Rushton, S. K., Harris, J. M., Lloyd, M. R., \& Wann, J. P. (1998). Guidance of locomotion on 824 foot uses perceived target location rather than optic flow. Curr Biol, 8, 1191-1194.

825 Rushton, S. K., Wen, J., \& Allison, R. S. (2002). Egocentric direction and the visual 826 guidance of robot locomotion background, theory and implementation. Biologically 827 Motivated Computer Vision, Proceedings, 2525, 576-591. 
828 Schemmel, J., Bruderle, D., Grubl, A., Hock, M., Meier, K., \& Millner, S. (2010). A wafer829 scale neuromorphic hardware system for large-scale neural modeling. In Circuits and 830 Systems (ISCAS), Proceedings of 2010 IEEE International Symposium on (pp. 1947-1950).

831 Seubert, J., Humphreys, G. W., Muller, H. J., \& Gramann, K. (2008). Straight after the turn: 832 the role of the parietal lobes in egocentric space processing. Neurocase, 14, 204-219.

833 Simoncelli, E. P., \& Heeger, D. J. (1998). A model of neuronal responses in visual area MT. 834 Vision Research, 38, 743-761.

835 Srinivasa, N., \& Cruz-Albrecht, J. M. (2012). Neuromorphic Adaptive Plastic Scalable 836 Electronics Analog Learning Systems. Ieee Pulse, 3, 51-56.

837 Srinivasan, M. V., \& Zhang, S. W. (1997). Visual control of honeybee flight. EXS, 84, 95838113.

839 Tanaka, K., Sugita, Y., Moriya, M., \& Saito, H. (1993). Analysis of object motion in the 840 ventral part of the medial superior temporal area of the macaque visual cortex. Journal of 841 Neurophysiology, 69, 128-142.

842 Wall, M. B., \& Smith, A. T. (2008). The representation of egomotion in the human brain. 843 Curr Biol, 18, 191-194.

844 Warren, W. H., Jr., Kay, B. A., Zosh, W. D., Duchon, A. P., \& Sahuc, S. (2001). Optic flow is 845 used to control human walking. Nat Neurosci, 4, 213-216.

846 Wen, B., \& Boahen, K. (2009). A Silicon Cochlea With Active Coupling. Ieee Transactions 847 on Biomedical Circuits and Systems, 3, 444-455.

848 Wilkie, R., \& Wann, J. (2003). Controlling steering and judging heading: retinal flow, 849 visual direction, and extraretinal information. J Exp Psychol Hum Percept Perform, 29, 363850378.

851 Xiao, D. K., Raiguel, S., Marcar, V., \& Orban, G. A. (1997). The spatial distribution of the 852 antagonistic surround of MT/V5 neurons. Cereb Cortex, 7, 662-677.

853

854 


\section{Figure captions}

Figure 1: "Le Carl" Android based robot, which was constructed from the chassis of an

$857 \mathrm{R} / \mathrm{C}$ car. The task of the robot was to navigate to a visually salient target (bright yellow 858 foam ball) while avoiding an obstacle (blue recycle bin) along the way. The robot's position

859 throughout the task was recorded by an overhead camera that tracked the position of the 860 green marker.

861

Figure 2: Technical setup. An Android app (ABR client) was used to record 320x240 864 made of two processing streams: an obstacle component responsible for inferring the 865 relative position and size of nearby obstacles by means of motion discontinuities, and a goal component responsible for inferring the relative position and size of a goal object by

867 means of color blob detection. These streams were then fused in a model of the posterior 868 parietal cortex to generate steering commands that were sent back to the ABR platform.

Figure 3: Schematic of the spatial receptive fields in the network. V1 neurons pooled retinal afferents and computed directional responses according to the motion energy

872 model (Simoncelli \& Heeger, 1998). The receptive fields of MT neurons had a circular

873 center preferring motion in a particular direction, surrounded by a region preferring 874 motion in the anti-preferred direction, implemented as a difference of Gaussians. PPC 875 neurons computed a net vector response weighted by the firing rates of MT neurons.

Figure 4: Camera setup. (A) The robot's initial view of the scene from the onboard Android phone. (B) View of an overhead camera that tracked the green marker attached to 879 the robot. (C) Birds-eye view image of the scene, obtained via perspective transformation 880 from the image shown in (B). The robot's location in each frame was inferred from the 881 location of the marker, which in turn was determined using color blob detection.

883 Figure 5: Behavior paths of the robot (colored solid lines) around a single obstacle 884 (recycle bin, ' $\mathrm{O}$ ') towards a visually salient goal (yellow foam ball, ' $\mathrm{X}$ ') for five different 
885 scene geometries, compared to "ground truth" (black dashed lines) obtained from the 886 behavioral model by Fajen and Warren (2003). Results are shown for steering with V1 887 (blue) as well as for steering with MT (red). Solid lines are the robot's mean path averaged 888 over five trials, and the shaded regions correspond to the standard deviation.

Figure 6: Rasterplot of neuronal activity for (a) V1 and (b) MT recorded in a single trial where the obstacle was 3 meters away and offset by 8 degrees (red colored line in Figure 892 5). A dot represents an action potential from a simulated neuron. For the sake of 893 visualization, only every eighth neuron in the population is shown over the time course of 894 six seconds that included both the obstacle avoidance and the goal approach. Neurons are 895 organized according to their direction selectivity, where neurons with ID 0-299 were most selective to rightward motion $\left(0^{\circ}\right)$, IDs 300-599 mapped to upward-rightward motion at $89745^{\circ}$, IDs $600-899$ mapped to upward motion at $90^{\circ}$, IDs $900-1199$ mapped to $135^{\circ}$, IDs 898 1200-1499 mapped to $180^{\circ}$, IDs 1500-1799 mapped to $225^{\circ}$, IDs 1800-2099 mapped to $899270^{\circ}$, and IDs $2100-2299$ mapped to $315^{\circ}$. (A) shows the spike trains generated from a 900 Poisson distribution with mean firing rate what the linear filter response is. Average 901 activity in the population was $18.6 \pm 15.44 \mathrm{~Hz}$. (B) shows the spike trains of Izhikevich 902 neurons in MT. Average activity in the population was $5.74 \pm 8.98 \mathrm{~Hz}$.

904 Figure 7: Overall network activity and corresponding behavioral output during obstacle 905 avoidance in a single trial. Each Panel summarizes the processing of a single visual input 906 frame, which corresponded to a time interval of $50 \mathrm{~ms}$. The indicated time intervals are 907 aligned with the neuronal traces presented in Figure 6. A frame received at time $t-50 \mathrm{~ms}$ 908 led to a motor response at time $t$. Neural activity during these $50 \mathrm{~ms}$ is illustrated as an 909 optic flow field, overlaid on visual input, for both Poisson spike generators in V1 and 910 Izhikevich spiking neurons in MT (population vector). Please note that vector length does 911 not correspond to velocity as is the case in a conventional optic flow field, but instead 912 indicates the "confidence" of the direction estimate. For the sake of clarity, only every 913 fourth pixel location is visualized. The resulting turning rate, $\dot{\theta}$, which was mapped to a 914 PWM signal for the robot's servos, is illustrated using a sliding bar, where the position of 
915 the triangle indicates the sign and magnitude of the turning rate. The small horizontal line

916 indicates $\dot{\theta}=0$. Note that the turning rate depended both on an obstacle term (from optic

917 flow) and a goal term (from color blob detection).

918

919 Figure 8: Overall network activity and corresponding behavioral output during goal920 directed steering in a single trial. Each Panel summarizes the processing of a single visual 921 input frame, which corresponded to a time interval of $50 \mathrm{~ms}$. The indicated time intervals 922 are aligned with the neuronal traces presented in Figure 6. A frame received at time $t-$ $92350 \mathrm{~ms}$ led to a motor response at time $t$. Neural activity during these $50 \mathrm{~ms}$ is illustrated as 924 an optic flow field, overlaid on visual input, for both Poisson spike generators in V1 and 925 Izhikevich spiking neurons in MT (population vector). Please note that vector length does 926 not correspond to velocity as is the case in a conventional optic flow field, but instead 927 indicates the "confidence" of the direction estimate. For the sake of clarity, only every

928 fourth pixel location is visualized. The resulting turning rate, $\dot{\theta}$, which was mapped to a 929 PWM signal for the robot's servos, is illustrated using a sliding bar, where the position of 930 the triangle indicates the sign and magnitude of the turning rate. The small horizontal line

931 indicates $\dot{\theta}=0$. Note that the turning rate depended both on an obstacle term (from optic 932 flow) and a goal term (from color blob detection). 


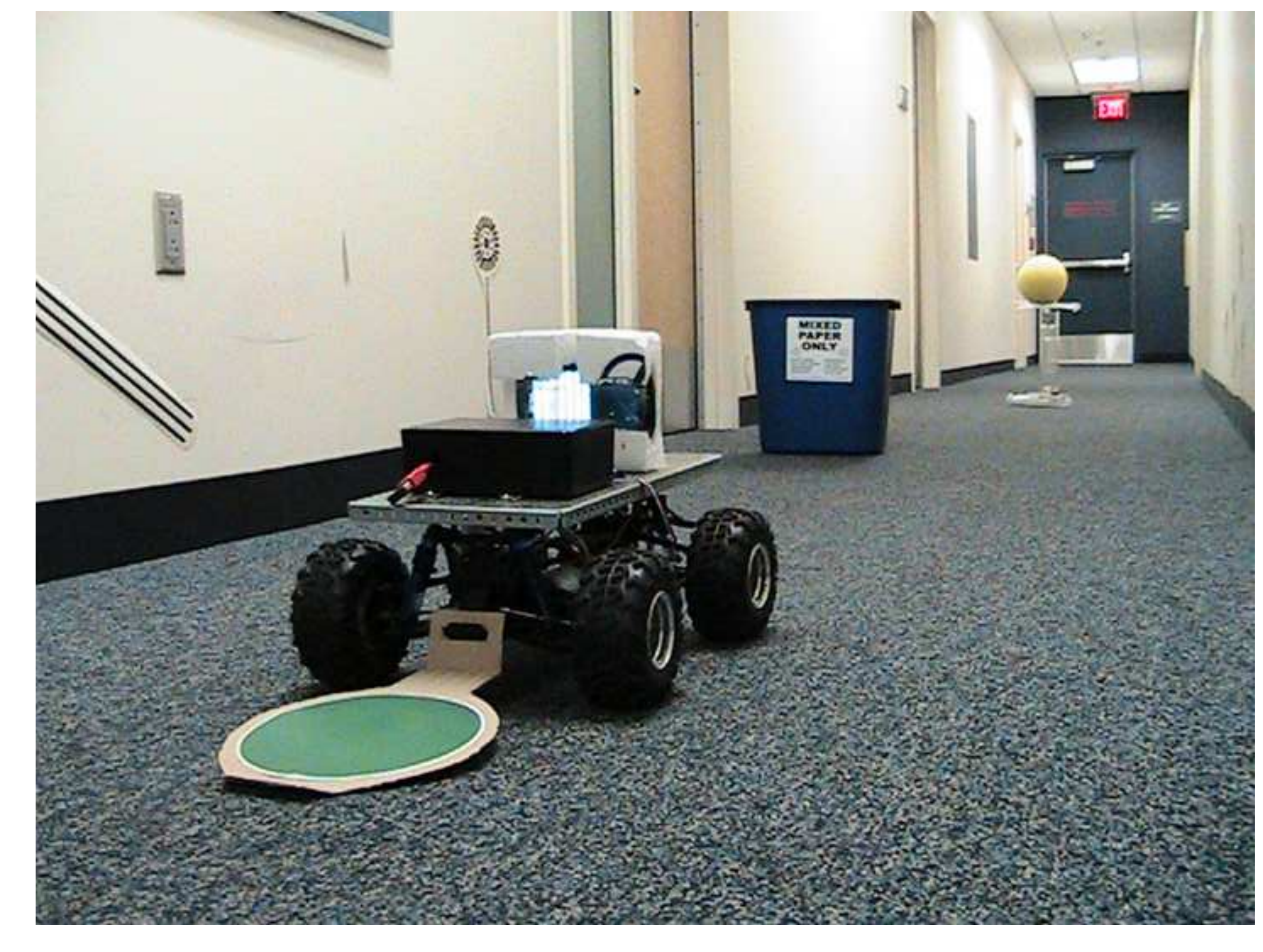

, 


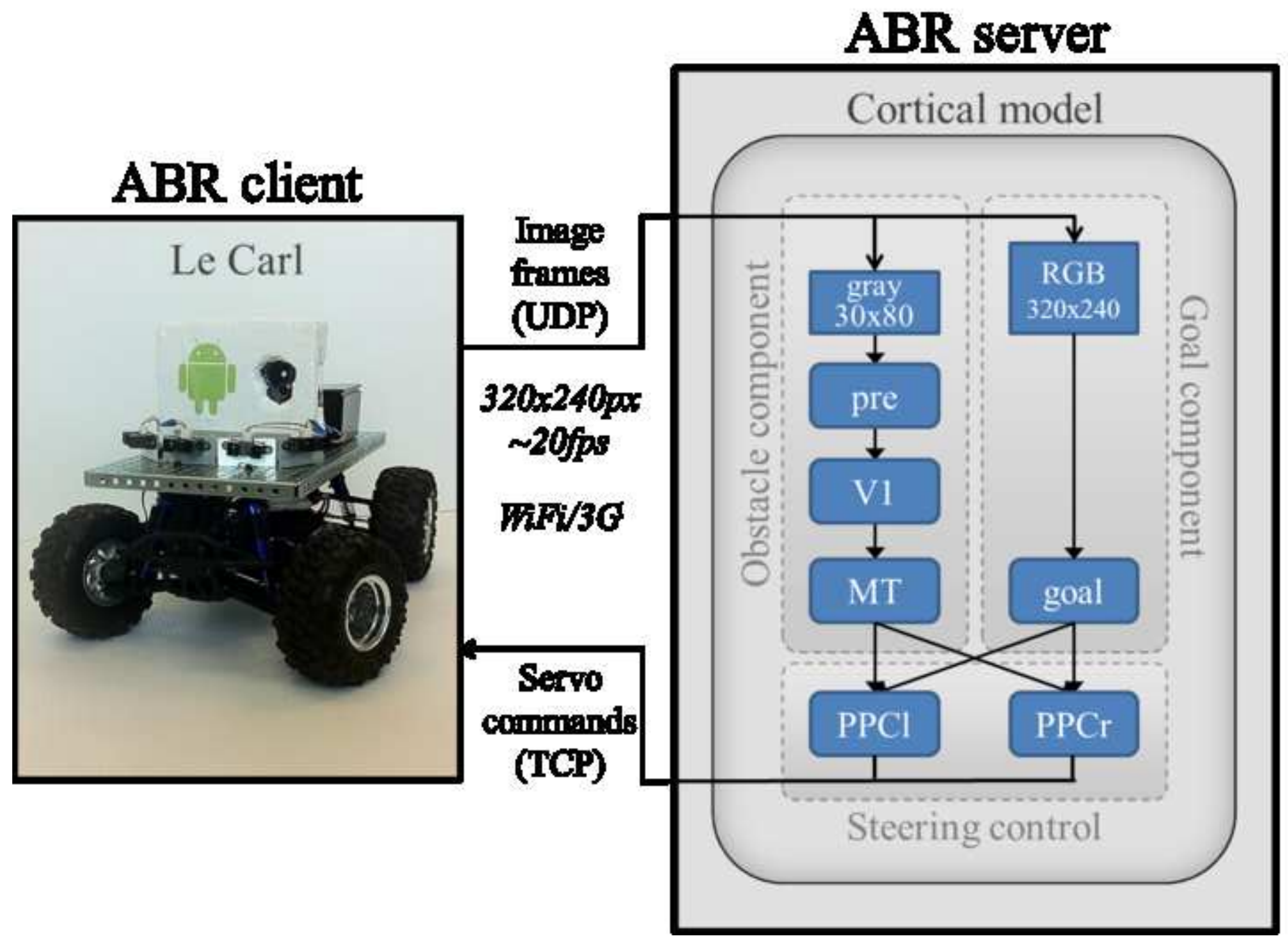

\section{ABR server}

Cortical model

ABR client inge frames (UDP)

$20 x 240 p x$

\%

WFF/3G

Servo commands (TCP)

\section{Steering control}


Retina $\rightarrow$ V1 V1 $\rightarrow$ MT

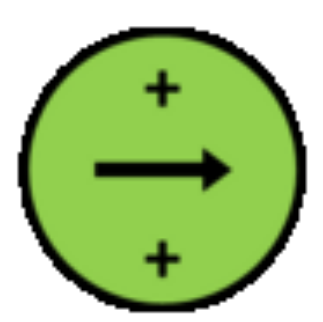

Local pooling

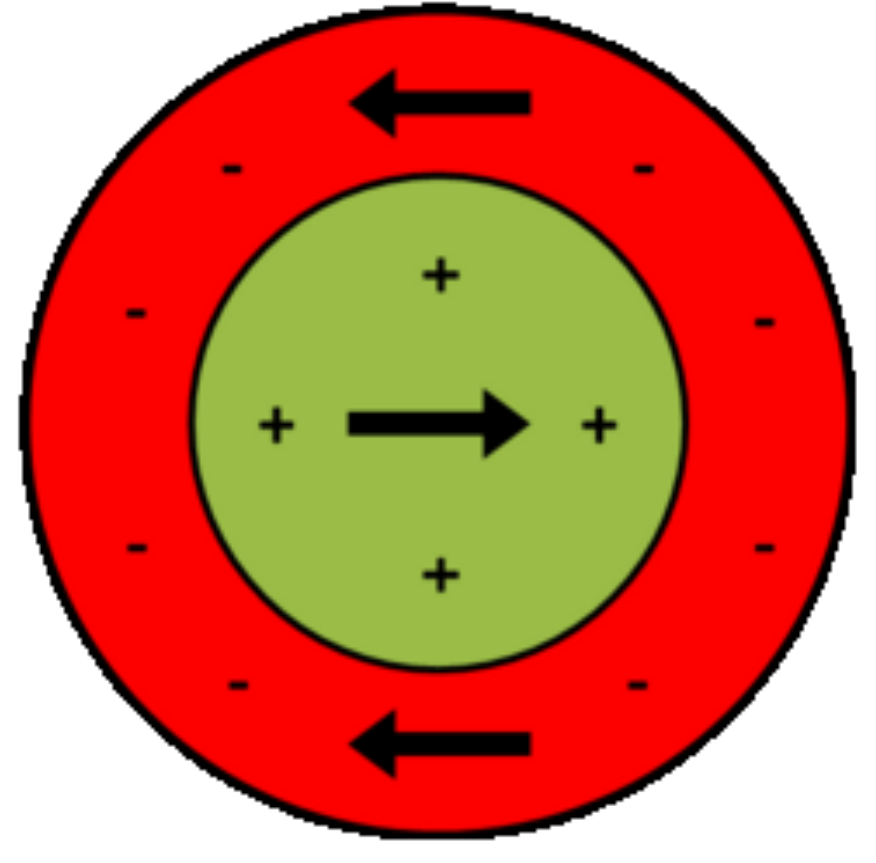

Center-surround
MT $\rightarrow$ PPC

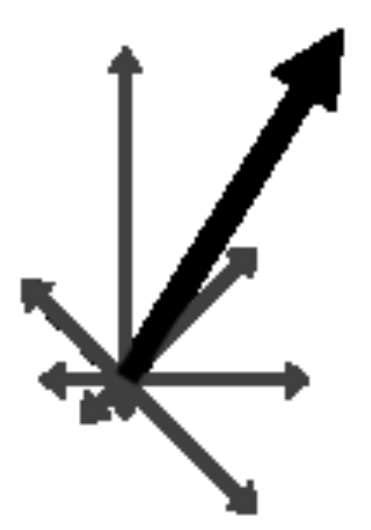

Vector sum 
A

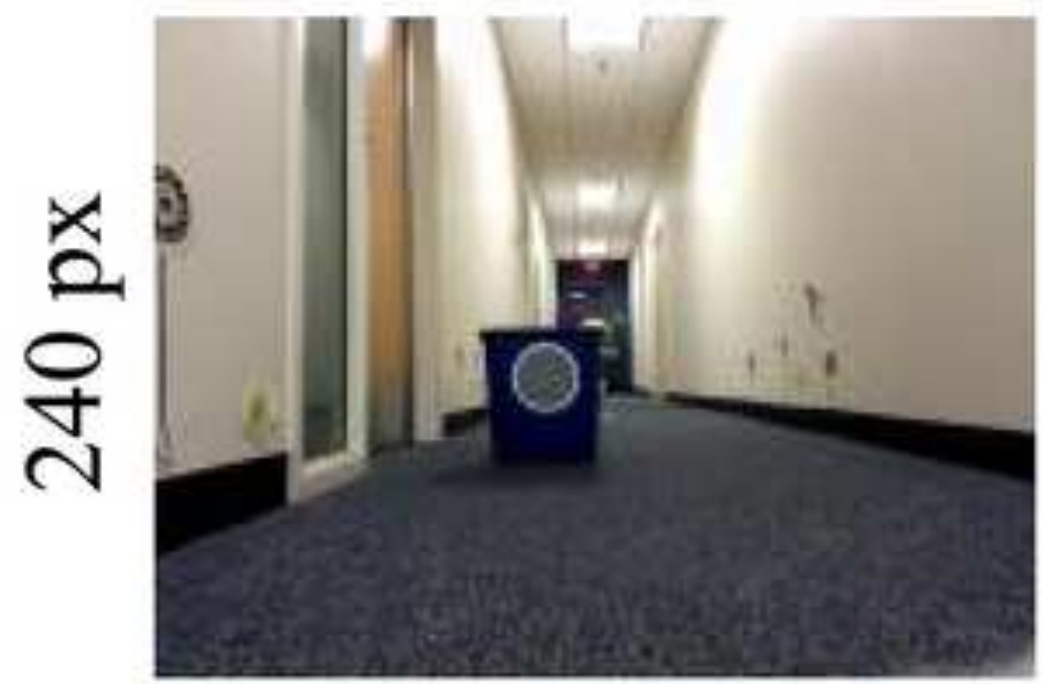

320 px

B

$\mathrm{C}$

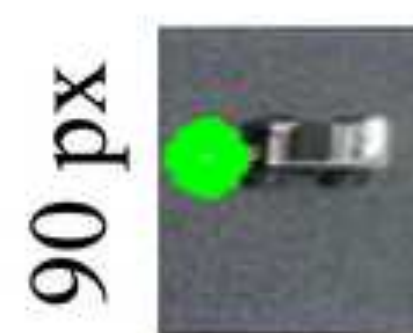 \\ 各붕}

320 px

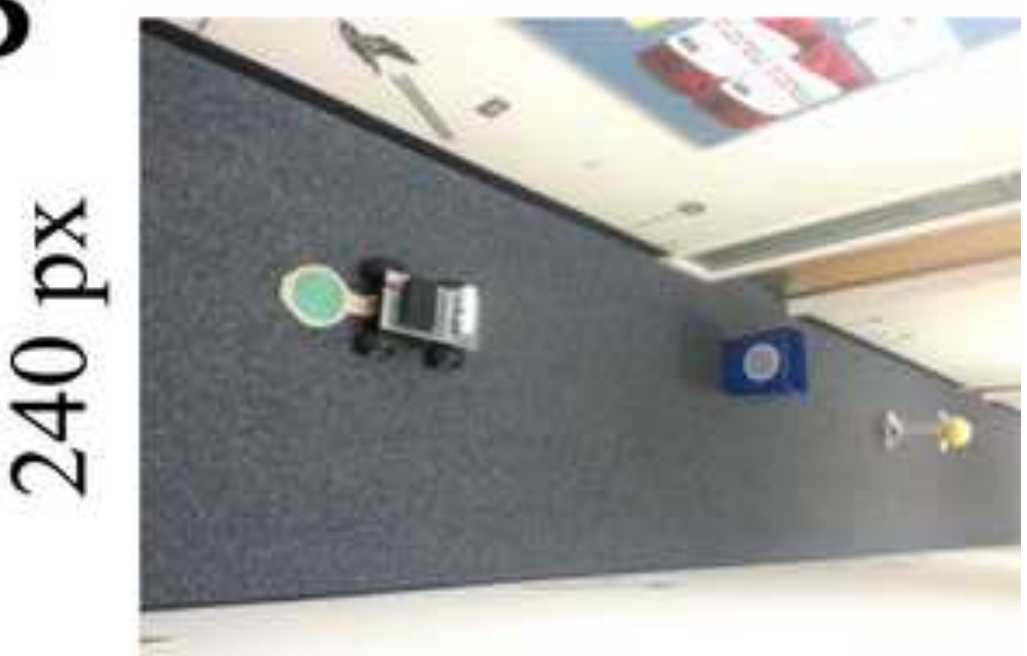

\section{0 px}




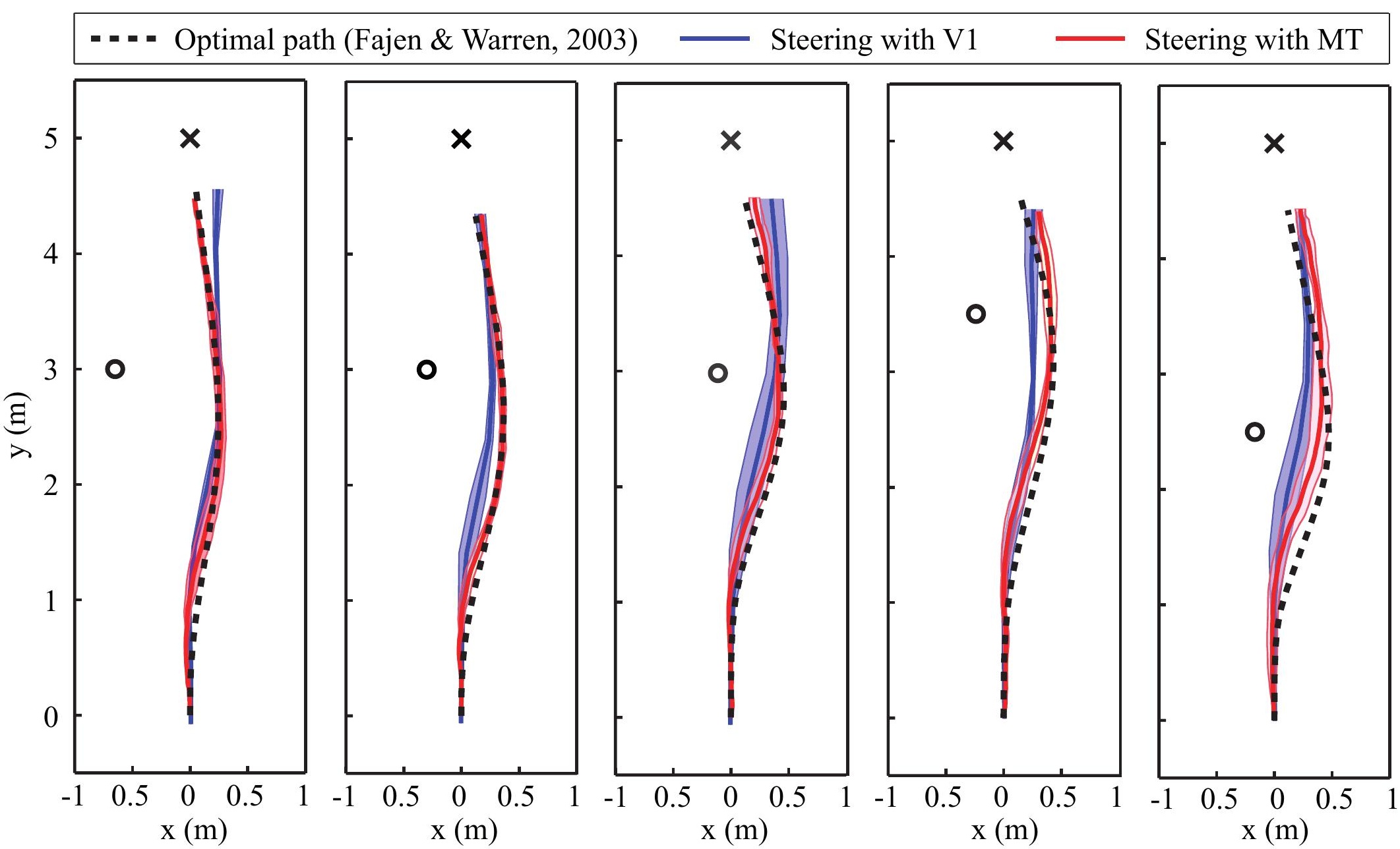


A
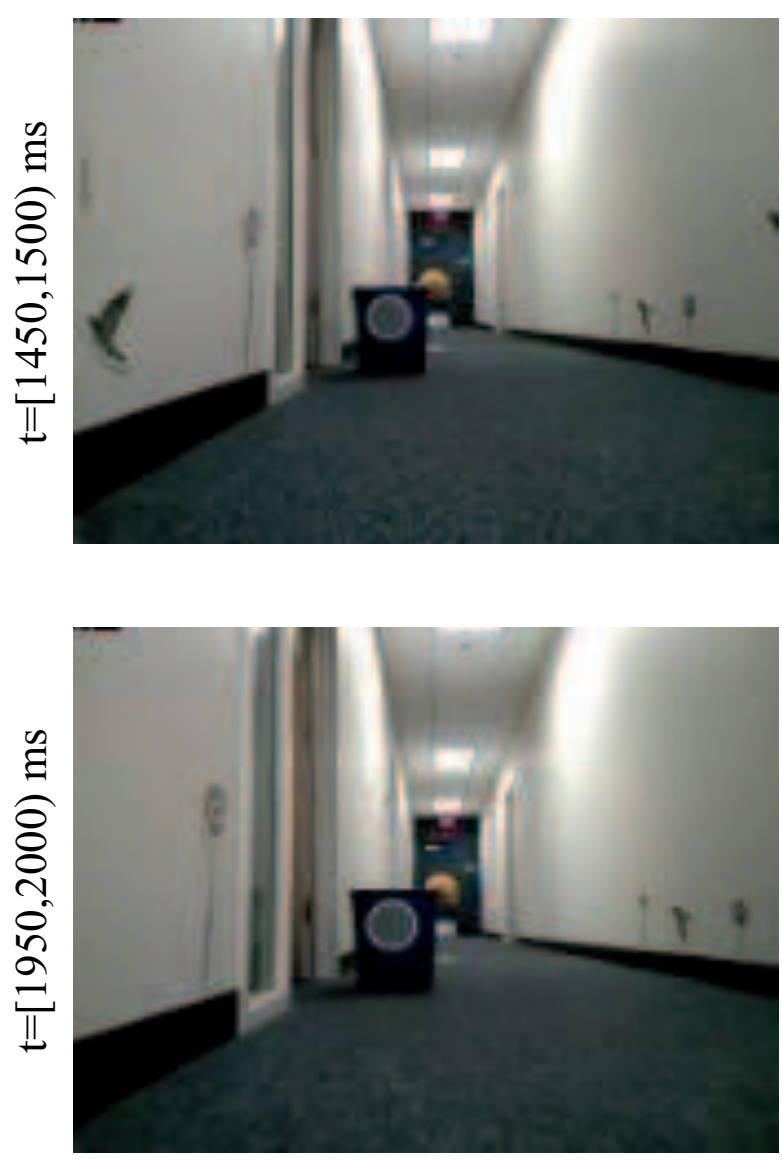

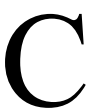
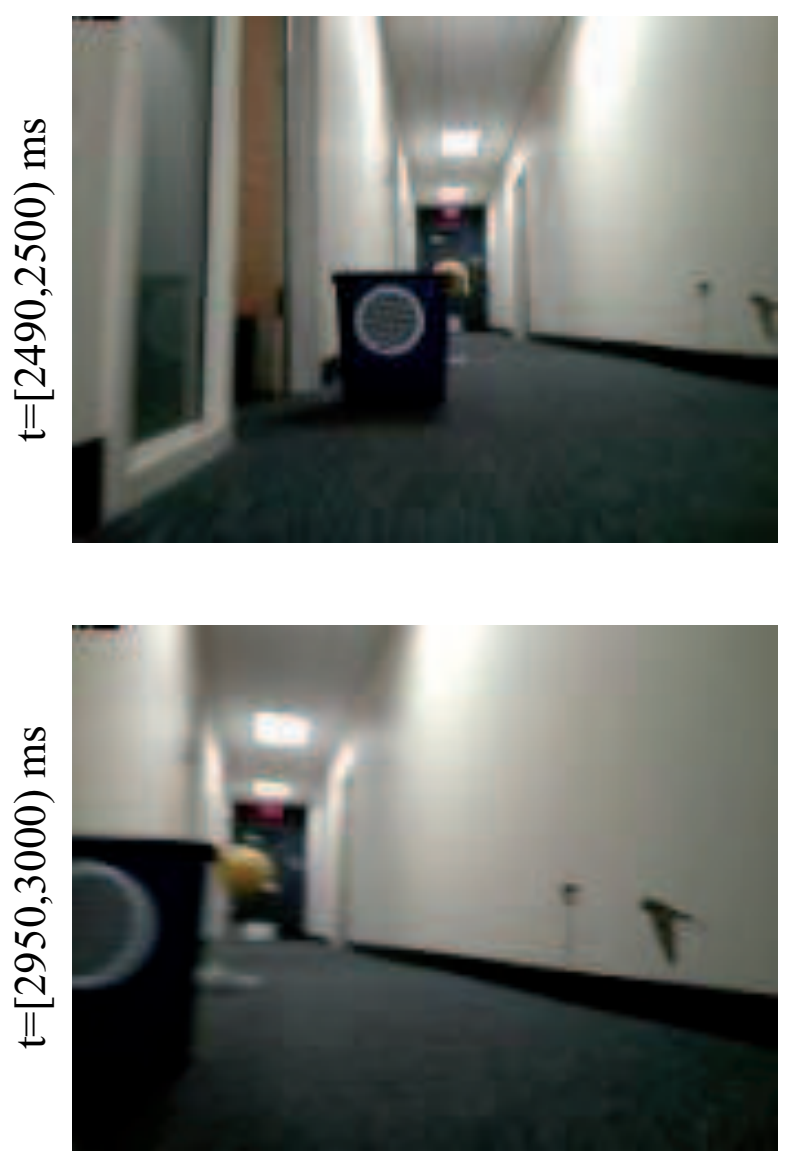
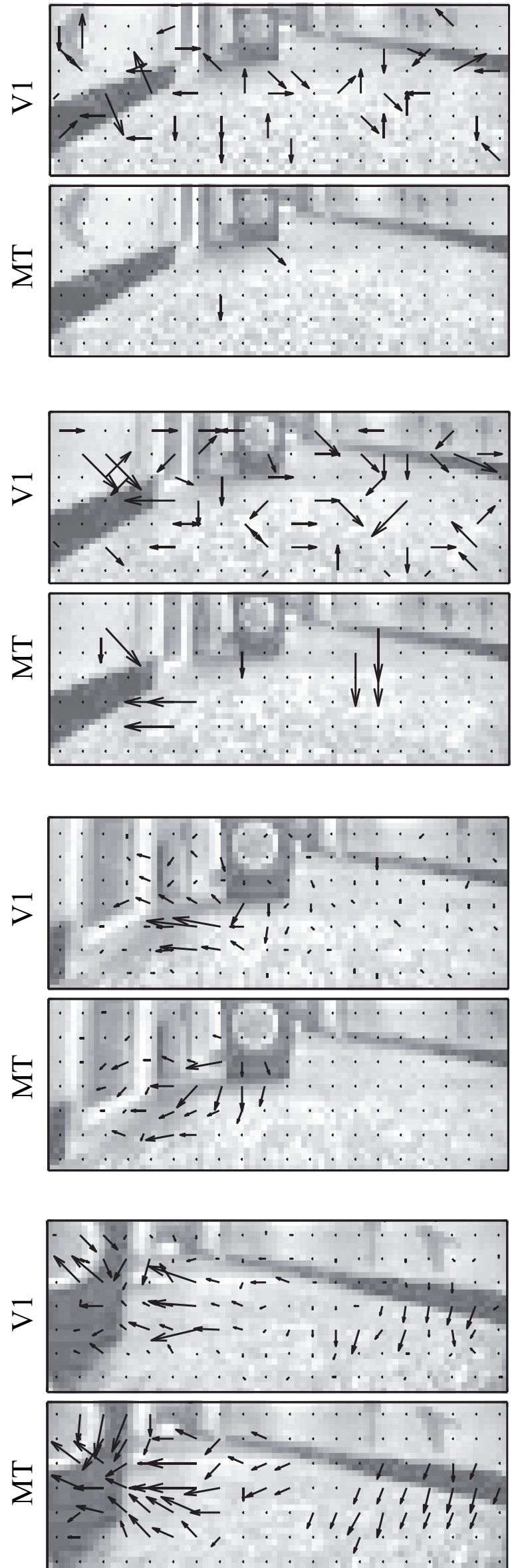

(3)

RIGHT

LEFT

RIGHT

0
0
0
2

LEFT

RIGHT

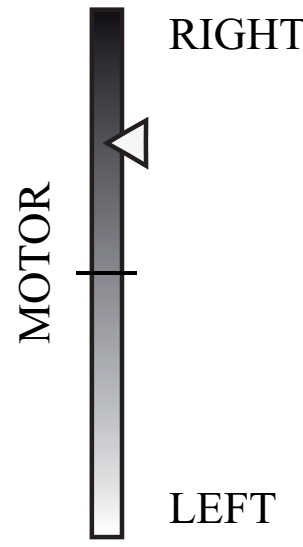

RIGHT

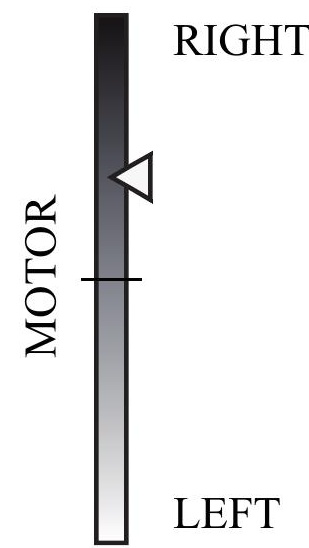


A
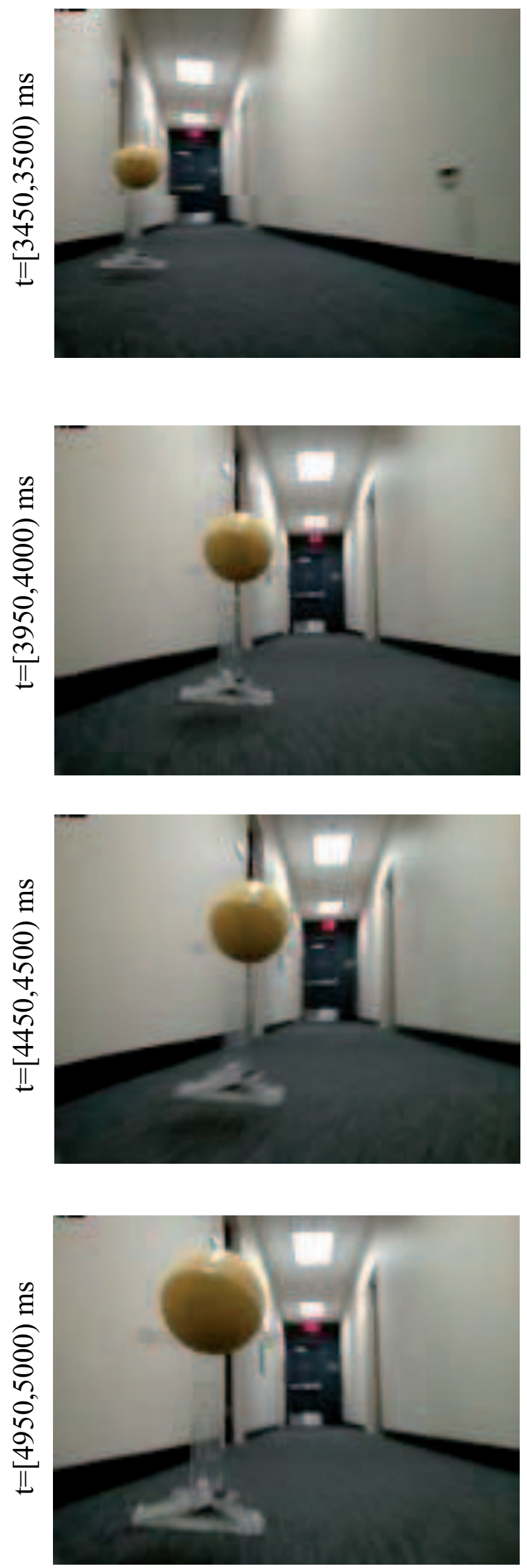
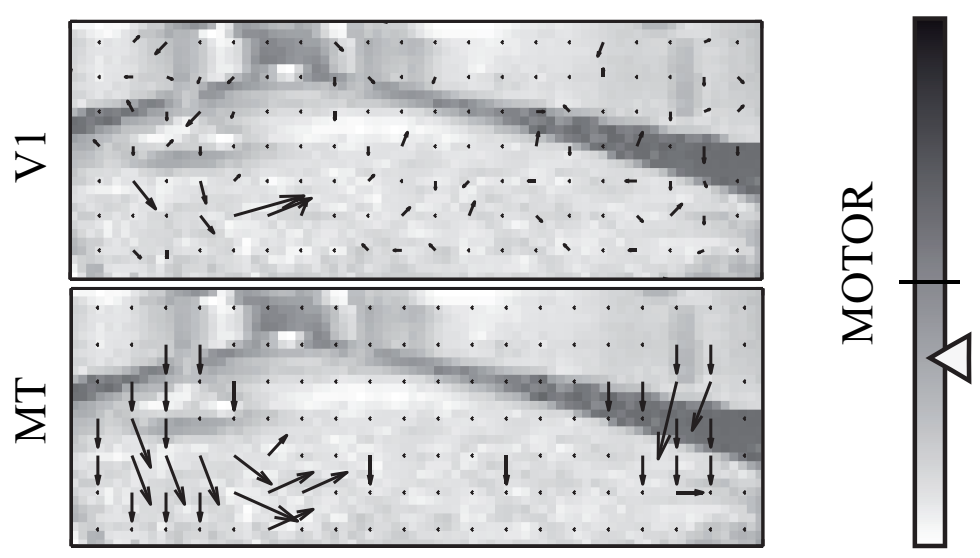

RIGHT

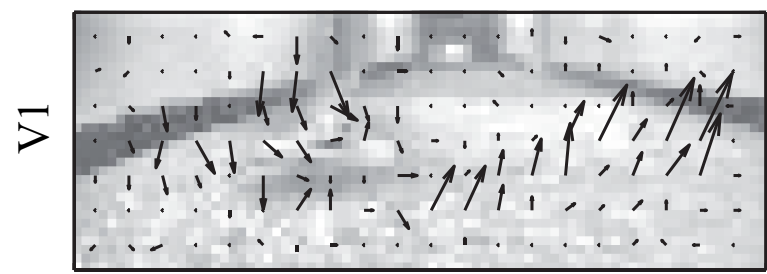

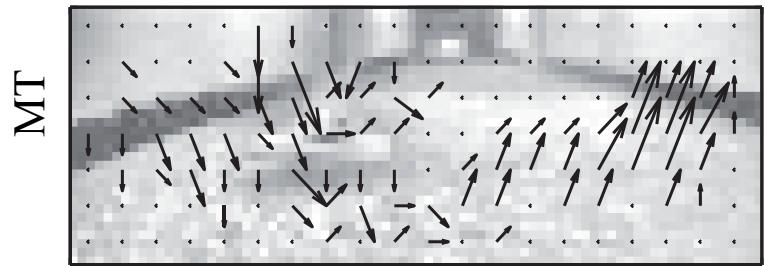

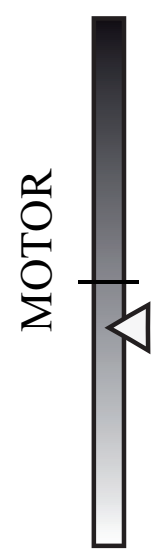

RIGHT

LEFT
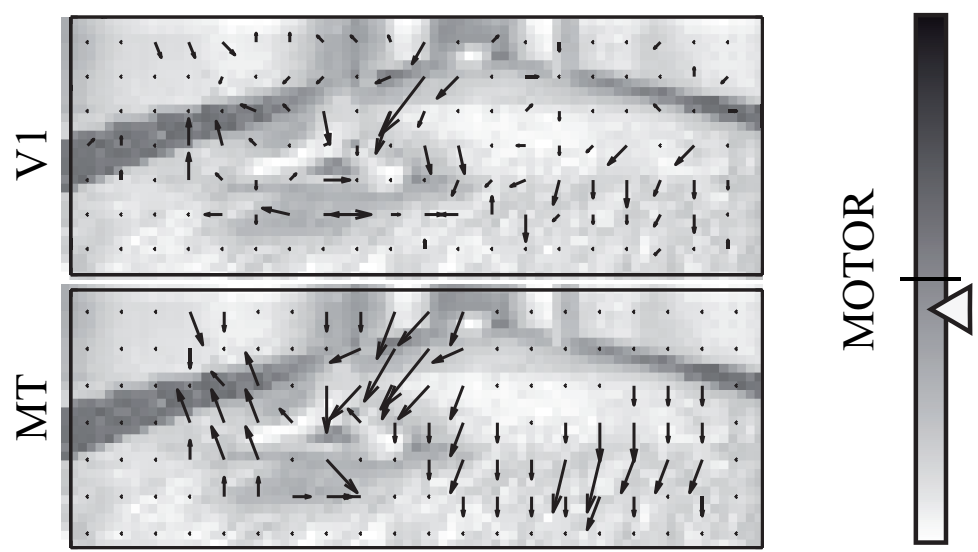

RIGHT

LEFT
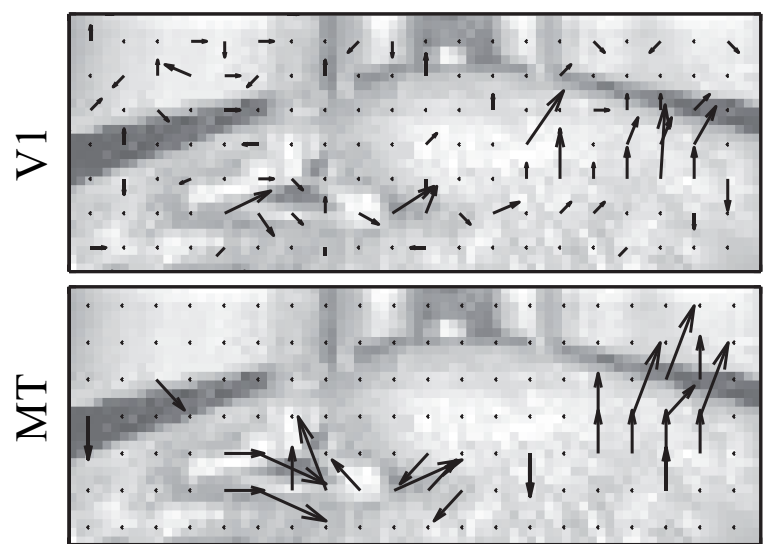

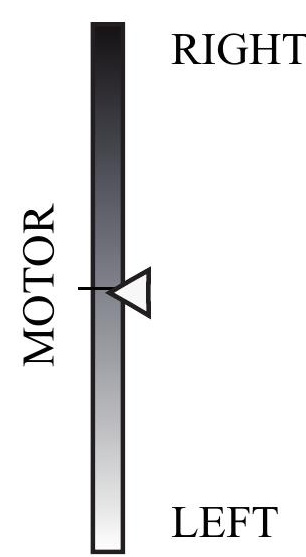

